\title{
Characterization of a Digital Camera as an Absolute Tristimulus Colorimeter
}

\author{
Francisco Martínez-Verdú凶 \\ Departamento Interuniversitario de Óptica, Universidad de Alicante, Alicante, Spain
}

J. Pujol

Centro de Desarrollo de Sensores, Instrumentación y Sistemas (CD6), Departamento de Óptica y Optometría, Universidad Politécnica de Cataluña, Barcelona, Spain

P. Capilla

Departamento de Óptica, Universidad de Valencia, Valencia, Spain

\begin{abstract}
An algorithm is proposed for the spectral and colorimetric characterization of digital still cameras (DSC) which allows them to be used as tele-colorimeters with CIE-XYZ color output, in $\mathrm{cd} / \mathrm{m}^{2}$. The spectral characterization consists in the calculation of the color-matching functions from the previously measured spectral sensitivities. The colorimetric characterization consists in transforming the raw RGB digital data into absolute tristimulus values CIE-XYZ (in $\mathrm{cd} / \mathrm{m}^{2}$ ) under variable and unknown spectroradiometric conditions. Thus, in the first stage, a gray balance was applied over the raw RGB digital data to convert them into RGB relative colorimetric values. In the second stage, an algorithm of luminance adaptation versus lens aperture was inserted in the basic colorimetric profile. Capturing the ColorChecker chart under different light sources, and comparing the estimated XYZ data according to the developed color model in relation to the measured XYZ data $\left(\right.$ in $\left.\mathrm{cd} / \mathrm{m}^{2}\right) \mathrm{using}$ a telespectroradiometer, we verified that the proposed characterization model may be broken down into two portions. Firstly, there is the basic colorimetric profile in combination with the new luminance adaptation algorithm. Secondly, there is the linear correction term due only to the mismatch of the color matching functions of the camera. Although the linear color correction term works relatively well, despite the imposed initial conditions (unknown spectral content of the scene), the separation of the proposed characterization model into two portions (raw and corrected performance) would allow the future comparison of various commercial cameras.
\end{abstract}

Journal of Imaging Science and Technology 47: 279-295 (2003)

\section{Introduction}

From image capture to acceptable color reproduction in an output device display (softcopy) or printer (hardcopy), the generic imaging chain ${ }^{1,2}$ or workflow for digital photography consists of several successive stages (Fig. 1), each of which is described in terms of the state of the image. At first, the image consists of the scene being photographed as seen through the zoom lens of the electronic still picture camera. Next, the image sensor (CCD or CMOS) and its associated electronics (internal gain, white balance, analog-digital converter) convert the optical image into a digital image representing the device dependent raw response of the camera. Without taking into account the aspects of color quantization and

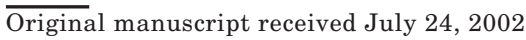

\section{A IS\&T Member}

Supplemental Materials-An Appendix can be found in color on the IS\&T website (www.imaging.org) for a period of no less than two years from the date of publication.

Color Plates 1-5 are printed in the color plate section of this issue, pp. 372-376.

C2003, IS\&T-The Society for Imaging Science and Technology conversion scaling, ${ }^{3}$ image compression and the data format of the file, ${ }^{4-6}$ the image is then transformed into a device independent representation $\left(\mathrm{CIE}^{-} \mathrm{XYZ}, \mathrm{CIE}^{-}\right.$ $\left.\mathrm{L}^{*} \mathrm{a} \mathrm{b}^{*}\right)$, which represents the scene itself, and not any input or output device. This color transformation (shown in italics in Fig. 1) between the raw RGB and CIE-XYZ spaces is the subject of this work, as specified in the ISO 17321 standard..$^{7,8}$

At the center of the diagram, the image is color rendered, i.e., the image may undergo some transformations in order to compensate for the differences in the input and output viewing conditions (tone,${ }^{9}$ gamut mapping, ${ }^{10}$ etc.). These operations are therefore typically proprietary and irreversible. In the color rendering stage, the state of the image changes from input-referred to standard output-referred (sRGB, ${ }^{11}$ extended sRGB, ${ }^{11,12}$ ICC PCS, ${ }^{13}$ SWOP CMYK, ${ }^{14}$ etc.), but is still device independent. Next, the image is transformed to a device dependent representation, reversing the device color characterization model, so that the image can be downloaded in an output-ready state to a specific output device. A final viewable image is produced by this output device as either softcopy (displays: CRT, LCD, etc.) or hardcopy (color printers: ink jet, sublimation, offset, etc.). 


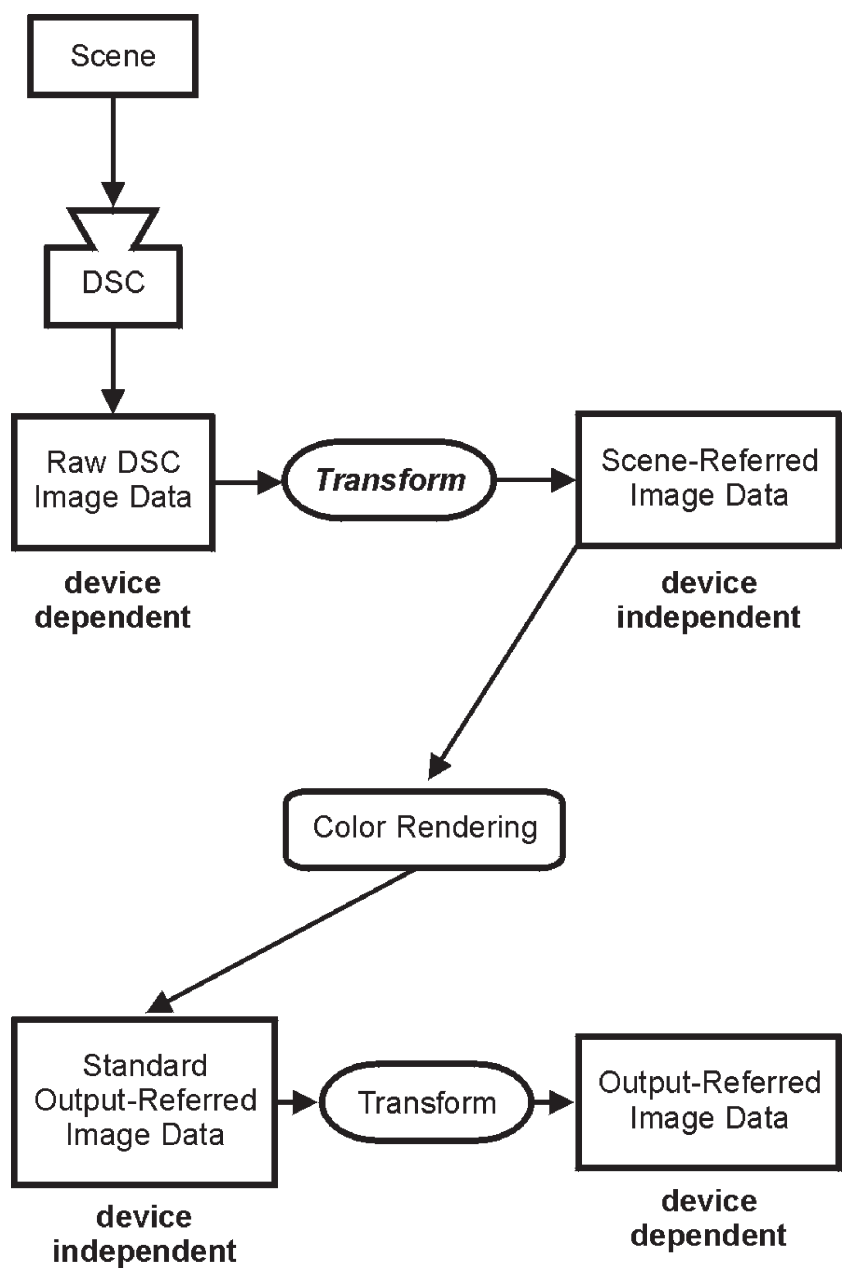

Figure 1. Imaging chain for digital photography.

\section{Selection of the Raw DSC-RGB Color Space}

When a digital image capture device (scanner or camera) is color characterized, it is necessary to take into account both the details of characterization and calibration. The color characterization model associated with a multimedia device consists of a reversible analytical model linking the digital RGB output levels of the device and the absolute tristimulus values XYZ (in $\left.\mathrm{cd} / \mathrm{m}^{2}\right)$ of the CIE standard observer. Even if the spectroradiometric conditions in a scene are fixed, there are a great variety of factors in an image capture device that can alter the RGB encoding of the same scene. The lens aperture or $\mathrm{f}$ number $N$ of the zoom-lens, the photosite integration time $t$ of the electronic shutter and the type of color architecture (3-CCDs, stripe color filter, etc.) of the image sensor can be called extrinsic factors because they act before the analog and optoelectronic image formation. On the other hand, the internal gain, the electronic white balance and the digitalization parameters are intrinsic factors because they mediate between the analog and digital stages of image formation. In principle, any variation of the exposure level $H$ over the image sensor due to the lens aperture $N$ (the illuminance scale exposure series) changes the raw digital still camera (DSC) image data but not the raw DSC-RGB space associated with its spectral sensitivities. The same should occur with the time scale exposure series. Therefore, a color characterization model of digital image capture devices should include at least the influence of the lens aperture $N$ or the photosite integration time $t$. In this study, we present a general color characterization model which includes the influence of the lens aperture or f-number $N$ of the zoom-lens using a luminance adaptation algorithm.

In order to work with raw or input-referred data as the ISO 17321 standard $^{7,8}$ claims, the only permitted image processing operations include color pixel reconstruction (demosaicking ${ }^{15,16}$ ), flare removal and electronic white balance. However, the image processing operations that render the image data, i.e., the tone mapping due to the change in dynamic range of the scene's luminance, so that they become output-referred (ready to display or to print), disqualify the data. Moreover, if the electronic white balance is changed, the raw DSC-RGB space will also be modified because the spectral sensitivities are changed correspondingly. Therefore, this change should be reflected in the color characterization model of the device, although it is difficult to implement.

\section{Basic Colorimetric Profile for DSCs}

Therefore, the color characterization of digital image capture devices consists in calculating the colorimetric profile between RGB device space and the CIE-1931 XYZ space associated with the same color stimulus under uncontrolled color and intensity illumination conditions. For this reason, the digital image capture device should perform as an absolute tele-colorimeter because the color output data should be in $\mathrm{cd} / \mathrm{m}^{2}$. That is to say, the color device should be simultaneously a colorimeter and a luminance meter (Fig. 2). Thus, from the raw DSC image data, i.e., digital output levels $D O L_{k}$ where $k=\mathrm{R}$, $\mathrm{G}$, B color channels, we can obtain the absolute tristimulus values CIE-XYZ (in $\mathrm{cd} / \mathrm{m}^{2}$ ) as with a telespectroradiometer (TSRM in Fig. 2; Eq. 1). Using a vector space notation, ${ }^{17-20}$ these values are as follows:

$$
\mathbf{t}_{\mathbf{X Y Z}}=\left[\begin{array}{c}
X \\
Y \\
Z
\end{array}\right]=K_{m} \mathbf{T}_{\mathbf{X Y Z}}{ }^{\mathrm{t}} \cdot \mathbf{c}
$$

where $K_{m}$ is $683 \mathrm{~lm} / \mathrm{W}, \mathbf{T}_{\mathbf{X Y Z}}=[\bar{x} \bar{y} \bar{z}]_{41 \times 3}$ are the colormatching functions in matrix format from 380 to 780 $\mathrm{nm}$ at $10 \mathrm{~nm}$ steps, and $\mathbf{c}=\operatorname{diag}(\mathbf{L}) \cdot \rho$ is the color stimulus, resulting from the spectral reflectance $\rho$ of the object and the light source or illuminant $\mathbf{L}$ in $\mathrm{W} / \mathrm{sr} \cdot \mathrm{m}^{2}$. In what follows, $\mathbf{A}^{\mathrm{t}}$ denotes the transpose of the matrix $\mathbf{A}$, and $\operatorname{diag}(\mathbf{x})$ the diagonal matrix from the vector $\mathbf{x}$.

If a digital still camera (DSC) were to perform simultaneously as a colorimeter and a photometer, it would combine these characteristics to become a telespectroradiometer-colorimeter (TSRM in Fig. 2), with the advantage that it could then simultaneously measure the entire visual field. Such a color device would have numerous industrial and scientific applications: spatial and color characterization of the displays, micro-colorimetry, comparison of camera models according to the raw color reproduction, color appearance models, color management, etc.

The basic colorimetric profile ${ }^{18}$ is a $3 \times 3$ matrix $\mathbf{M}$ which should associate the RGB relative colorimetric values or $\mathbf{t}_{\text {RGB }}$ with the relative tristimulus values $\mathbf{t}_{\mathbf{X Y Z}}$ normalized to the equal-energy stimulus or adapted white, $\mathbf{E}=[1,1, \ldots, 1]^{\mathrm{t}}$ (Eq. 2), and not to the adopted white, according to the terminology used in ISO 12231 standard. ${ }^{1,7,8}$ The estimated relative tristimulus values $\mathrm{XYZ}$ (in prime) should therefore be as follows: 


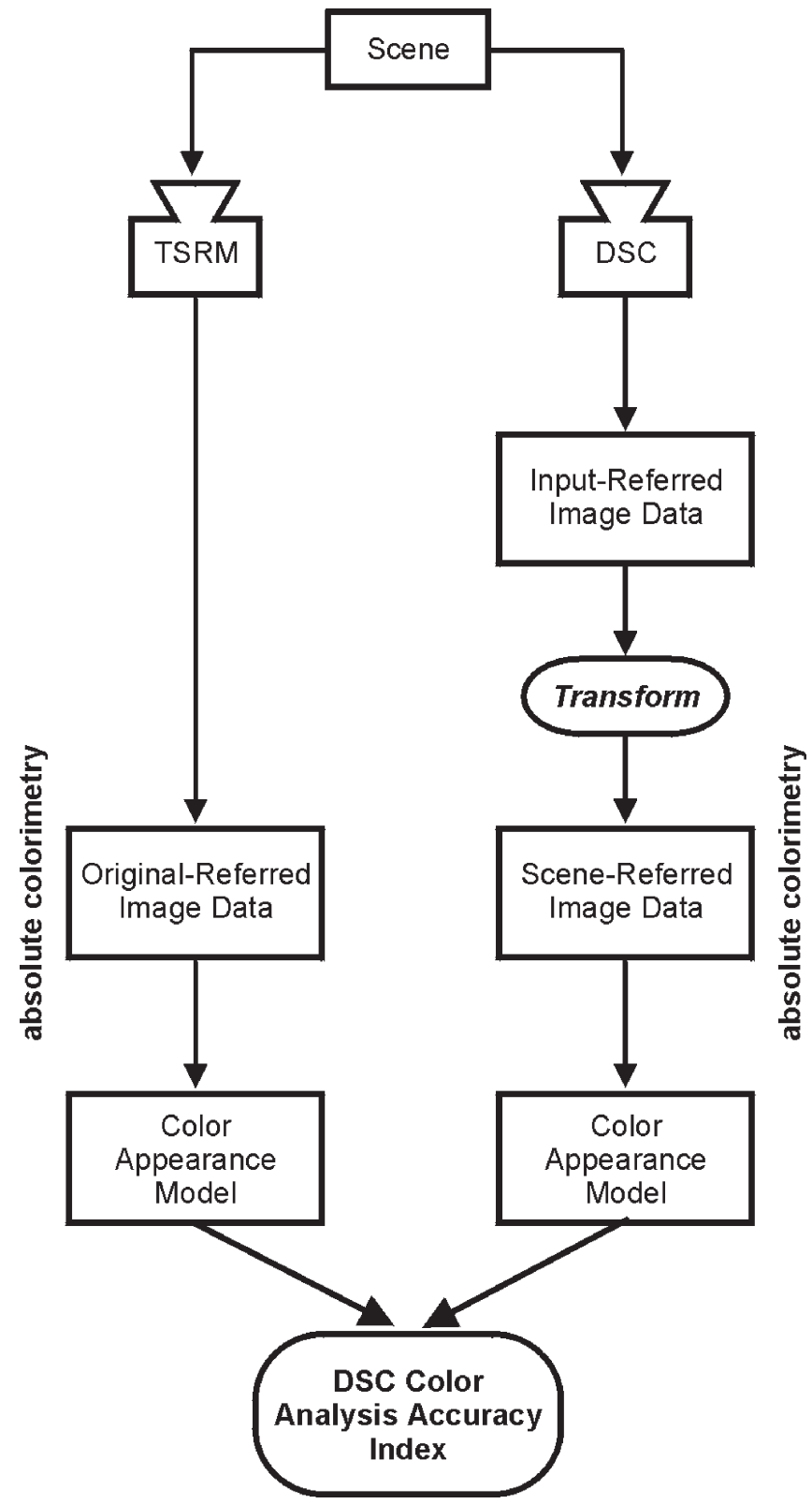

Figure 2. Colorimetric chain for obtaining the DSC color analysis accuracy index.

$$
\hat{\mathbf{t}}_{\mathbf{X Y Z}}^{\prime}=\mathbf{M} \cdot \mathbf{t}_{\mathbf{R G B}}^{\prime} \quad \text { with } \quad \mathbf{T}_{\mathbf{X Y Z}}{ }^{\mathrm{t}}=\mathbf{M} \cdot \mathbf{T}_{\mathbf{R G B}}{ }^{\mathrm{t}}
$$

If our purpose is to obtain an absolute estimation, in $\mathrm{cd} / \mathrm{m}^{2}$, of the tristimulus values XYZ, we must link a group of luminances associated with the equal-energy stimulus or illuminant $\mathbf{E}$ with the corresponding RGB data of the color device. This relationship is the camera opto-electronic conversion function ${ }^{21}$ (OECF). For any scale exposure series (varying the lens aperture or fnumber $N$ of the zoom lens or the photosite integration time $t$ ), the $O E C F s$ are described as follows:

$$
\mathbf{t}_{\mathbf{R G B}}^{\prime}=\left[\begin{array}{c}
R \\
G \\
B
\end{array}\right]=\left[\begin{array}{l}
O E C F_{R}(L) \\
O E C F_{G}(L) \\
O E C F_{B}(L)
\end{array}\right]
$$

where $L$ is the object luminance (in $\mathrm{cd} / \mathrm{m}^{2}$ ).

Therefore, the relative colorimetric profile is obtained by reversing the camera $O E C F s$ as follows:

$$
\hat{\mathbf{t}}_{\mathbf{X Y Z}}^{\prime}=\frac{1}{L_{\mathrm{E}}} \mathbf{M} \cdot\left[\begin{array}{l}
O E C F_{R}{ }^{-1}(R) \\
O E C F_{G}{ }^{-1}(G) \\
O E C F_{B}{ }^{-1}(B)
\end{array}\right]
$$

where $L_{\mathrm{E}}$ is the luminance of the adapted white ${ }^{1,7,8}$ or the perfect white diffuser in the scene.

In principle, the choice of the reference white in the color transformation between the two color spaces is crucial. If we wish to use a digital still camera as a photocolorimeter, it is essential that we select the equal-energy illuminant or adapted white $\mathbf{E}$ as the reference white, and not the adopted white of the scene, which is associated with the electronic white balance according to the chromaticity of the light source or illuminant. However, the ISO 14524 standard $^{21}$ proposes an algorithm for obtaining the photometric function $O E C F$ with fixed lens aperture $N$ or photosite integration time $t$ and any illumination different from illuminant $\mathbf{E}$. This standard is used in turn in the ISO 17321-2 standard $^{8}$ in order to obtain the relative colorimetric profile associated with a digital still camera (DSC).

Among the available options for obtaining the basic colorimetric profile, $\mathbf{M}$, there are two main categories, depending on the optimization method: least squares or regression methods, ${ }^{22}$ and principal components ${ }^{23}$ using algebraic and geometric methods with convex sets. Considering whether it is necessary to have a previous knowledge of the spectral content of the scene, the above mathematical methods can be divided into three general categories ${ }^{8,24}$ :

1. Maximum Ignorance: unknown and variable colorant behavior and unknown and variable illumination behavior: only the spectral sensitivities of the color device are known. If $\mathbf{T}_{\mathbf{X Y Z}}$ are the color-matching functions of the CIE standard observer and $\mathbf{T}_{\mathbf{R G B}}$ are the color-matching functions of the color device, the basic colorimetric profile, $\mathbf{M}$, is obtained as follows:

$$
\mathbf{M}=\mathbf{T}_{\mathbf{X Y Z}}{ }^{\mathrm{t}} \cdot \mathbf{T}_{\mathbf{R G B}} \cdot\left(\mathbf{T}_{\mathbf{R G B}}{ }^{\mathrm{t}} \cdot \mathbf{T}_{\mathbf{R G B}}\right)^{-1}
$$

If the gray balance is not correct, i.e., if $\mathbf{M} \cdot[1,1,1]^{\mathrm{t}}$ is not equal to $[1,1,1]^{\mathrm{t}}$, Finlayson and Drew ${ }^{22}$ propose a maximum ignorance constrained least squares regression preserving the white point vector which is denoted by MaxIgWPPLS. If we want to preserve the chromaticity of other colors, this technique allows this option, offering a color correction of sorts.

2. A priori knowledge: statistically expected colorant behavior and limited and fixed illumination behavior, i.e., it is assumed that the previous spectral content of the training set of objects and illuminants can be decomposed into $x$ basis functions. If $\mathbf{S}$, with $n_{\lambda}$ rows and $n$ columns, denotes the training set of spectral reflectances (the 24 patches of the GretagMacbeth ColorChecker chart), and $\mathbf{K}=\mathbf{S} \cdot \mathbf{S}^{\mathrm{t}}$ is the autocorrelation matrix ${ }^{22}$ of $\mathbf{S}$, the basic colorimetric profile $\mathbf{M}$ is obtained as follows:

$$
\mathbf{M}=\mathbf{T}_{\mathbf{X Y Z}}{ }^{\mathrm{t}} \cdot \mathbf{K} \cdot \mathbf{T}_{\mathbf{R G B}} \cdot\left(\mathbf{T}_{\mathbf{R G B}}^{\mathrm{t}} \cdot \mathbf{K} \cdot \mathbf{T}_{\mathbf{R G B}}\right)^{-1}
$$


The fact that the number $x$ of the basis functions is larger than the conventional three RGB color channels of the digital image capture device has given rise to various lines of research. These cover such aspects as multispectral imaging (new image capture devices, ${ }^{25,26}$ optimized design of scanning color filters, ${ }^{27-30}$ etc.), the simulation of illumination changes ${ }^{20,31}$ and the recovery of the spectral reflectance of an object. ${ }^{31-33}$

3. Minimum Ignorance: known and fixed colorant behavior and illumination. This is the typical procedure for scanners, using color targets according to ISO $12641^{34}$ and generating three-dimensional look-up tables $^{35}$ or least squares polynomial models, ${ }^{22,36-38}$ or imposing any optical model for colorant and media behavior, ${ }^{39}$ although it is possible to achieve similar results without a color target. ${ }^{40,41}$

In the latter two techniques or categories, it is customary to use the same samples for both training and testing under well-fixed spectroradiometric conditions, so the total color difference $\Delta \mathrm{E}$ metrics are excellent. In this work we will present a color characterization model which performs independently of previous knowledge, the intensity and chromaticity of the light source, and the spectral content of the objects in the scene. The training set will be the empirical determination of the colormatching functions ${ }^{42} \mathbf{T}_{\mathbf{R G B}}$ of the color device. The test set will be the ColorChecker chart under three real light sources (not CIE illuminants) inside a non-standard light box. The spectral characteristics of the light sources and the patches of the color chart will be not used.

According to these guidelines, summarized in Table I, we will also show which of the four basic colorimetric profile options $\mathbf{M}$ performs best. It seems, a priori, that the basic colorimetric profiles with previous spectral knowledge of the scene (LSAPK and PC) should yield the best results. This analysis ultimately involves determining which color error metrics are best for comparing the scene-referred image data with the original-referred image data in absolute colorimetry format, i.e., in $\mathrm{cd} / \mathrm{m}^{2}$ (Fig. 2). This problem, referred to as the DSC color analysis accuracy index, deserves to be analyzed separately in the following section of this introduction.

\section{DSC Color Analysis Accuracy Index}

Like the ISO 17321-2 standard, ${ }^{8}$ we are interested in methods for deriving transformations from digital camera data in order to estimate scene colorimetry (Fig. 2), i.e., the color transform between the input-referred image data (raw RGB data) and the scene-referred image data (estimated CIE-XYZ data). This task should include an error minimization method (color correction) and a metric for quantifying the errors in scene colorimetry estimation. This means a comparison of the scene-referred image data with the original-referred image data (measured CIE-XYZ data).

A preliminary approximation would be to use only the spectral sensitivities of the color device, quantifying the common volume between the sub-vectorial spaces associated with the two sets of color-matching functions $\mathbf{T}_{\mathbf{R G B}}$ and $\mathbf{T}_{\mathbf{x y z}}$. This approach was proposed by Neugebauer as the quality factor $q$ in 1956 and has been extended with new quality factors ${ }^{43-49}$ or figures of color merit for image capture devices, both of which minimize the mean-squared error metric in CIE-XYZ or CIE-L*a*b* spaces. However, these algorithms do not take into account a direct comparison between the original-referred
TABLE I. Basic Colorimetric Profiles for a Digital Image Capture Device.

\begin{tabular}{ccc}
\hline No. & Label & Description \\
\hline$M_{1}$ & MaxlgLS & Maximum ignorance by least-squares regression \\
$M_{2}$ & MaxlgWPPLS & $\begin{array}{r}\text { Maximum ignormance by least-squares regression } \\
\text { Preserving the white point vector }\end{array}$ \\
$M_{3}$ & LSAPK & Least-squares regression with a-priori knowledge \\
$M_{4}$ & PC & Principal components \\
\hline
\end{tabular}

and the scene-referred image data. To do this, it is necessary to translate the problem into a color appearance model format. Only if we use the absolute colorimetric format from the beginning can we work with realistic color appearance models. ${ }^{50}$ If we prefer to use the relative colorimetry format, the simplest color appearance model available is the CIE-L*a*b* space. In this work we will use only the CIE-L*a*b* space as first approximation to obtain the DSC color analysis accuracy index. Nevertheless, we are studying alternative procedures for a subsequent work to this article with more complex color appearance models that operate with absolute tristimulus values (in $\mathrm{cd} / \mathrm{m}^{2}$ ) as input.

Digital image capture devices (scanners and cameras) are pseudo-colorimeters because their spectral sensitivities, due to engineering and technological conditions, ${ }^{24,51,52}$ are not exact linear combinations of the CIE-XYZ color matching functions (Luther condition ${ }^{53-}$ ${ }^{55}$ ). Therefore, systematic color reproduction errors will unavoidably appear. In spite of this, decades of experience in color photography and television have shown that acceptable color reproduction for typical scenes can be achieved using non-colorimetric RGB sensitivity curves. Unfortunately, some current color correction techniques ${ }^{56,57}$ make it very difficult to know the true quality level or accuracy index of the color reproduction of these color devices: for example, whether in the raw state or performance (with raw RGB space) the red gamut is encoded too yellow or the blue gamut is encoded too dark. In theory, because these color deviations are associated with systematic error sources, they could be compensated automatically. Nevertheless, we believe it would be very useful to also study the DSC color analysis accuracy index in the raw performance in order to compare various digital image capture devices (cameras and scanners).

In short, a general color characterization of any digital still camera will be presented in this work in order to convert this color device into an absolute tele-colorimeter. This procedure for the development of a new color measurement instrument has been patented (see pending codes $\left.{ }^{58,59}\right)$. The proposed algorithm is divided into two parts: spectral and colorimetric characterizations. The spectral characterization algorithm ${ }^{42}$ has already been published in this journal, but part of the results will be necessary to solve the colorimetric characterization algorithm. Let $\mathbf{t}_{\mathbf{X Y Z}}$ be the original-referred image data (Eq. 1; Fig. 2), as obtained by a telespectrocolorimeter, and let be the scene-referred image data from the proposed colorimetric profile (Eq. 4) with luminance adaptation. Our aim is to test this reproduction model by capturing the ColorChecker chart under three light sources, selectively varying the f-number $N$ of the zoom lens of the camera, and making no assumptions regarding the scene's spectral radiance correlation statistics (maximum ignorance). We will show that the original-referred image data of the unclipped colors can be estimated by regression using a linear color correc- 
tion model, consisting of a tristimulus vector $\mathbf{A}_{\mathbf{C}}$ and the scaling diagonal matrix $\mathbf{B}_{\mathbf{C}}$. Since raw RGB digital data will be used from the beginning and the Luther condition is not fulfilled for our camera, the raw colorimetric profile will be redefined as $\mathbf{B}_{\mathbf{C}}$. and the tristimulus vector $\mathbf{A}_{\mathbf{C}}$ will be the only correction term due to the mismatch of the color matching functions of the camera.

\section{Materials and Methods}

There are basically two experimental set-ups: one for the spectral characterization (monochromator set-up ${ }^{42}$ ) and one for the colorimetric characterization (viewing booth set-up).

Our digital image capture device consisted of a Sony DXC-930P 3CCD-RGB camera with a zoom lens (model VCL-712BXEA) connected to a Matrox MVP-AT 850 frame grabber, inserted into a PC unit. With these components it was not necessary to apply demosaicking and compression operations because the camera architecture is of the 3-CCD type (with a dichroic prism block) and the images obtained from the frame grabber were in $512 \times 512$ format. Among the fixed initial conditions, which might alter the color output, we set the electronic white balance to $5600 \mathrm{~K}$ in manual menumode (offset value) and configured the gain and the offset of the analog-to-digital converter (ADC) to work with the raw response space. Therefore, according to the guidelines of the ISO $17321^{7,8}$ standard, raw RGB digital data are always used. In all exposure processes, the photosite integration time $t$ of the electronic shutter was fixed to offset value $t_{0}=20 \mathrm{~ms}$, so the exposure was determined by carefully varying the lens aperture $N$ of the zoom-lens.

Throughout the experiments, we will consider the spectral exposure $H(\lambda)$ (in joules) to be proportional to the spectral radiance $L_{e}(\lambda)$ of the object and the photosite integration time $t$, and inversely proportional to the fnumber $N$. With a centered capture of visible light, ${ }^{42}$ the spectral exposure $H(\lambda)$ can be described as follows:

$$
H(\lambda)=\alpha \frac{L_{e}(\lambda)}{N^{2}} t, \text { with } \alpha=\frac{\pi}{4} \frac{A_{\text {SENSOR }}}{\left(1+m_{\text {LENS }}\right)^{2}}
$$

where $A_{S E N S O R}$ is the irradiated image sensor and $m_{L E N S}$ is the lateral magnification of the zoom lens.

We will also assume that the reciprocity law-that identical values of spectral exposure yield identical responses even if the lens aperture $N$ or the exposure time $t$ change-is fulfilled ${ }^{60}$ in digital photography. This law regarding the exposure on the image sensor is not fulfilled in photochemical photography with either monochromatic or white light. However, some of our own experiments have shown that this exposure law is in fact fulfilled in digital photography using monochromatic light. Therefore, as discussed above, the variation of the f-number $N$ or the exposure time t does not mean that the raw DSC-RGB color space, or equivalently the spectral sensitivities of the color device, should change.

The only assumption we must make with regard to the basic optoelectronic performance and the physics of image capture is that the univariance principle holds (Eq. 8). If $n_{p e}(\lambda)$ is the number of spectral generated photoelectrons from the incident photon rate $n_{v}(\lambda)$, the total number will be $n_{p e}$ according to the univariance principle. In a simplified model of the final optoelectronic step between the spectral generated photoelectrons $n_{p e}(\lambda)$ and the spectral digital output levels $D O L(\lambda)$ by means of the optoelectronic conversion constant ${ }^{61,62}$ $K(\lambda)$, the total digital output level $D O L$ is:

$$
\begin{aligned}
n_{p e} & =\sum_{380 \mathrm{~nm}}^{780 \mathrm{~nm}} n_{p e}(\lambda) \Delta \lambda \\
& =\sum_{380 \mathrm{~nm}}^{780 \mathrm{~nm}} n_{v}(\lambda) Q E(\lambda) \Delta \lambda \\
D O L & =\left(2^{\text {bits }}-1\right) \sum_{380 \mathrm{~nm}}^{780 \mathrm{~nm}} N D O L(\lambda) \Delta \lambda \\
& =\left(2^{\text {bits }}-1\right) \sum_{380 \mathrm{~nm}}^{780 \mathrm{~nm}} H(\lambda) r(\lambda, H) \Delta \lambda
\end{aligned}
$$

where $Q E(\lambda)$ is the spectral quantum efficiency, or quantum version of the spectral responsitivity $r(\lambda, H)$ of the digital image capture device, and $N D O L$ is the normalized digital output level.

The first step in characterizing a digital image capture device as an absolute tristimulus colorimeter is the spectral characterization. This we did in a previous work. ${ }^{42}$ We proved that the univariance principle may be rewritten using the analytical expression of the optoelectronic conversion spectral functions (OECSFs) measured from the monochromator experiment as follows:

$$
\begin{aligned}
& D O L_{k}=\left(2^{\text {bits }}-1\right) \sum_{380 \mathrm{~nm}}^{780 \mathrm{~nm}}\left[a_{\lambda k}+\frac{b_{\lambda k}}{1+\exp \left(-\frac{H_{\lambda}-c_{\lambda k}}{d_{\lambda k}}\right)}\right] \Delta \lambda \\
& D O L_{k}=\left(2^{b i t s}-1\right) \sum_{380 \mathrm{~nm}}^{780 \mathrm{~nm}} H_{\lambda} r_{k}(\lambda, H) \Delta \lambda \\
& \Rightarrow r_{k}(\lambda, H)=\frac{a_{\lambda k}+\frac{b_{\lambda k}}{1+\exp \left(-\frac{H-c_{\lambda k}}{d_{\lambda k}}\right)}}{H}
\end{aligned}
$$

where $H_{\lambda}$ is the dense version of the spectral exposure (Eq. 7) and the values $\{a, b, c, d\}$ are the fitting parameters for each wavelength and color channel ( $k=\mathrm{R}, \mathrm{G}, \mathrm{B})$ of the sigmoid function that models the empirical relationship between the normalized digital output levels $N D O L_{k}$ and the spectral exposure $H(\lambda)$, denoted above as the opto-electronic conversion spectral function (OECSF).

The second step is colorimetric characterization. The necessary experimental procedure consists in capturing the GretagMacbeth ColorChecker chart under different light sources. The lamps used were a halogen lamp (INC), a metal halide lamp (HWL) and a daylight fluorescent lamp (DAY). To reduce flare, the walls and the base of the viewing booth were painted black and all the patches of the chart except one were masked (Color Plate 1, p. 372 ). A Photo Research PR-650 telespectroradiometer, in the same position as the camera, was used to measure the spectral radiances $L_{e}(\lambda)$ of all the chart patches under these light sources (Color Plates 2 through 4, pp. 372-373).

The conditions for the camera and frame grabber were the same as in the above experiment, that is, the elec- 


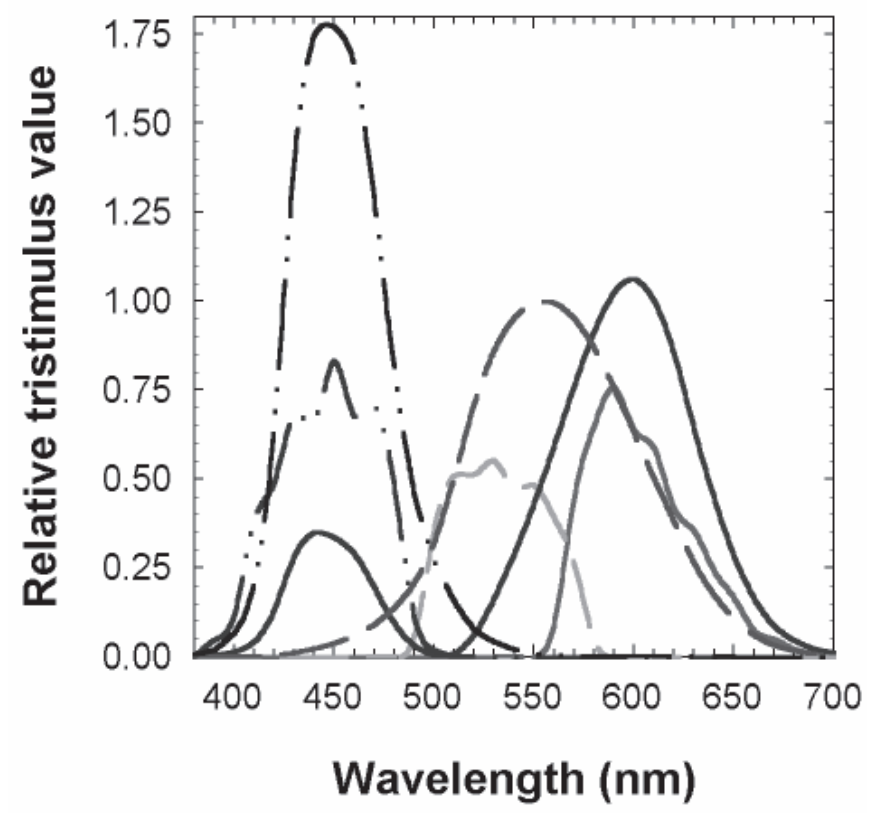

Figure 3. Color-matching pseudo-functions of the Sony DXC930P video camera plus a Matrox AT-850 frame grabber connected to the Standard Observer CIE-1931 XYZ. (Solid line: R or X channel; dashed line: G or Y channel; dash-dot-dot line: B or Z channel).

tronic white balance was fixed by control menu at 5600 $\mathrm{K}$. An exception to this is that the f-number $N$ of the zoom lens which was varied to maximize the dynamic range of raw digital data $(N=4$ for INC capture, $N=$ 5.6 for HWL and DAY captures). In spite of this, some colors were not encoded optimally because at least one of the three associated digital output levels was clipped, due to underexposure or saturation. From the possible 73 colors with the three captures, 53 useful colors remained. sRGB simulations of the ColorChecker chart under these light sources are also provided in Color Plates 2 through 4. Tables A1-A3 in the Appendix (Appendix can be found as Supplemental Material on the IS\&T website (www.imaging.org) for a period of no less than two years from the date of publication.) show the average digital output levels $D O L$ of the patches along with the corresponding absolute tristimulus values XYZ measured by the tele-spectroradiometer.

Due to the non-uniformity of vertical illumination inside the viewing booth and according to the relative colorimetric profile (Eq. 4), the luminances $L_{E}$ of a Halon reference white, used as adapted white, were measured by the tele-spectroradiometer in the 72 positions of the color chart under the three light sources. These photometric values are also incorporated in Tables A1-A3 in the Appendix.

\section{Colorimetric Characterization: Results and Discussion}

In a previous work ${ }^{42}$ we obtained the $\mathbf{T}_{\mathbf{R G B}}$ color matching functions (Fig. 3) for our digital image capture device. Basically, these spectral functions were the relative spectral sensitivities of the color device scaled by two factors (joint scaling and equal-energy white balance). With this information, the four basic colorimetric profiles are calculated below. The colorimetric or equal-energy white balance test (included in our previous work) is additional information necessary for solving the colori-

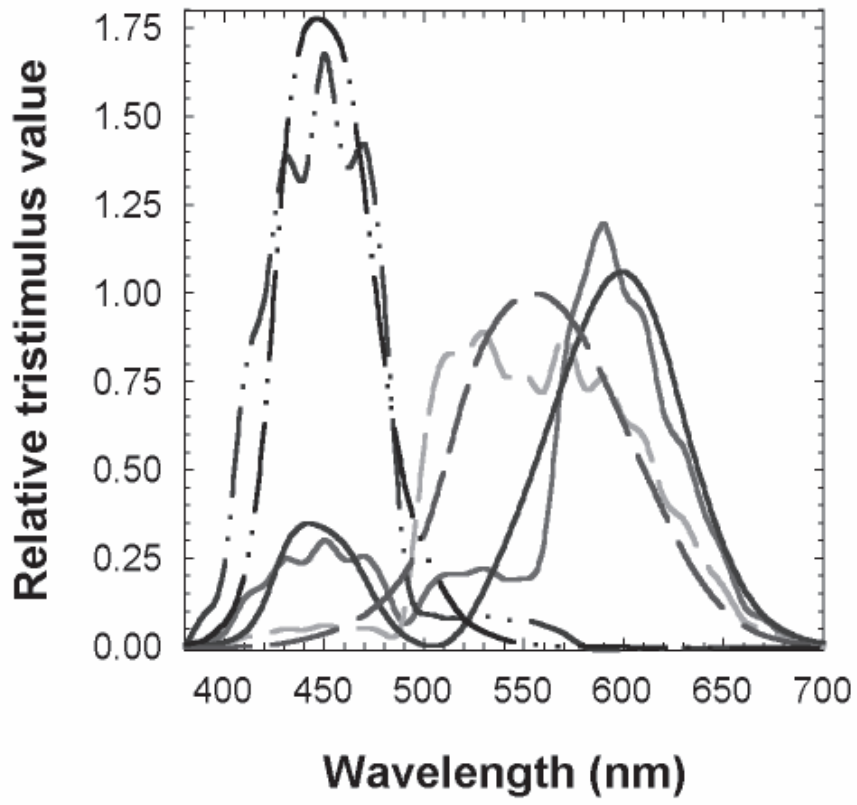

Figure 4. Estimated color-matching functions of the Standard Observer CIE-1931 XYZ from the basic colorimetric profile MaxIgLS and the color-matching pseudo-functions of our digital image capture device (Solid line: $\mathrm{R}$ or $\mathrm{X}$ channel; dashed line: G or Y channel; dash-dot-dot line: B or Z channel).

metric characterization. These are key data because they provide information regarding the ratio between the areas beneath the color matching functions associated with our color device. In the CIE-1931 XYZ space, this ratio is $1: 1: 1$ but, although the electronic white balance was fixed to $5600 \mathrm{~K}$ by the control menu, the ratio obtained was $b a l_{R}=0.8642, b a l_{G}=0.6839$ and $b a l_{B}=1$. One consequence of taking $b a l_{B}=1$ is that the $\mathbf{T}_{\mathbf{R G B}}$ color matching functions are scaled in relation to the blue channel and not in relation to the absolute scaling of the $\mathbf{T}_{\mathbf{X Y Z}}$ color matching functions. However, this disadvantage can be remedied by using color correction techniques such as those applied further on in this section.

We also present a reproduction model with luminance adaptation in order to estimate the $\mathbf{t}_{\mathbf{x y z}}$ absolute tristimulus values (in $\mathrm{cd} / \mathrm{m}^{2}$ ), such as they might be obtained by a tele-spectrocolorimeter. Afterwards, we will use CIE-L*a*b* color space as first approximation to quantify the prediction to true colorimetry of the scene (ColorChecker chart under some light sources).

\section{Calculation of the Basic Colorimetric Profiles}

According to Table I and Eq. 5, the basic colorimetric profile $\mathbf{M}_{1}$ with maximum ignorance using least squares regression is:

$$
\begin{aligned}
& \mathbf{M}_{1}=\mathbf{T}_{\mathbf{X Y Z}}{ }^{\mathrm{t}} \cdot \mathbf{T}_{\mathbf{R G B}} \cdot\left(\mathbf{T}_{\mathbf{R G B}}{ }^{\mathrm{t}} \cdot \mathbf{T}_{\mathbf{R G B}}\right)^{-1} \\
& =\left[\begin{array}{ccc}
1.5798 & 0.4016 & 0.3643 \\
1.0086 & 1.6157 & 0.0742 \\
-0.0107 & 0.1573 & 2.0189
\end{array}\right]
\end{aligned}
$$

Now we may graphically evaluate the fit by comparing the estimated $\mathbf{T}_{\mathbf{X Y Z}}\left(=\mathbf{T}_{\mathbf{R G B}} \cdot \mathbf{M}_{1}{ }^{\mathrm{t}}\right)$ color matching functions with the true $\mathbf{T}_{\mathbf{X Y Z}}$ values (Fig. 4). It is clear from this graph that the combined spectral functions $\mathbf{T}_{\mathbf{R G B}}$ are not 
strictly color matching functions, so it seems correct to denote them as color matching pseudo-functions.

The next option for the basic colorimetric profile $\mathbf{M}_{2}$ is associated with the preservation of the white point using a constrained least squares regression with maximum ignorance. Because $\mathbf{M}_{1} \cdot[1,1,1]^{\mathrm{t}}$ is not equal to $[1,1,1]^{\mathrm{t}}$, the MaxIgWPPLS algorithm ${ }^{22}$ can be applied as follows in order to obtain a new matrix $\mathbf{M}_{2}$ verifying that $\mathbf{M}_{2} \cdot[1,1,1]^{\mathrm{t}}$ is equal to $[1,1,1]^{\mathrm{t}}$ :

$$
\begin{aligned}
& \forall \text { color - stimulus } \mathbf{C}, \text { if } \mathbf{t}_{\mathbf{R G B}}^{\prime}(\mathbf{C})=\left[\begin{array}{l}
R \\
G \\
B
\end{array}\right] \neq\left[\begin{array}{l}
X \\
Y \\
Z
\end{array}\right]=\mathbf{t}_{\mathbf{X Y Z}}^{\prime}(\mathbf{C}) \\
& \Rightarrow \mathbf{M}_{\mathbf{2}}=\mathbf{D}_{\mathbf{2}}+\mathbf{E}_{\mathbf{2}}, \quad \text { where } \mathbf{D}_{\mathbf{2}}=\left[\begin{array}{ccc}
\frac{X}{R} & 0 & 0 \\
0 & \frac{Y}{G} & 0 \\
0 & 0 & \frac{Z}{B}
\end{array}\right]
\end{aligned}
$$

$\mathbf{E}_{\mathbf{2}}=\left(\mathbf{Z}_{\mathbf{2}} \cdot \mathbf{N}_{\mathbf{2}}\right)^{\mathrm{t}}$, satisfying $\mathbf{t}_{\mathbf{R G B}}{ }^{\mathrm{t}} \cdot \mathbf{E}_{\mathbf{2}}=0$,

with $\quad \mathbf{Z}_{\mathbf{2}}=\left[\begin{array}{ll}\boldsymbol{\sigma}_{\mathbf{1}} & \boldsymbol{\sigma}_{\mathbf{2}}\end{array}\right]_{3 x 2}, \quad \boldsymbol{\sigma}_{\mathbf{1}}{ }^{\mathrm{t}} \cdot \mathbf{t}_{\mathbf{R G B}}^{\prime}=\boldsymbol{\sigma}_{\mathbf{2}}{ }^{\mathrm{t}} \cdot \mathbf{t}_{\mathbf{R G B}}^{\prime}=0$

and $\mathbf{N}_{\mathbf{2}}=\left(\mathbf{Z}_{\mathbf{2}}{ }^{\mathrm{t}} \cdot \mathbf{T}_{\mathbf{R G B}}{ }^{\mathrm{t}} \cdot \mathbf{T}_{\mathbf{R G B}} \cdot \mathbf{Z}_{\mathbf{2}}\right)^{-1}$

$\cdot\left(\mathbf{Z}_{\mathbf{2}}{ }^{\mathrm{t}} \cdot \mathbf{T}_{\mathbf{R G B}}{ }^{\mathrm{t}} \cdot \mathbf{T}_{\mathbf{X Y Z}}-\mathbf{Z}_{\mathbf{2}}{ }^{\mathrm{t}} \cdot \mathbf{T}_{\mathbf{R G B}}{ }^{\mathrm{t}} \cdot \mathbf{T}_{\mathbf{R G B}} \cdot \mathbf{D}_{\mathbf{2}}\right)$

Because equal energy stimulus $\mathbf{E}$ verifies that $\mathbf{t}_{\mathbf{R G B}}=$ $\mathbf{t}_{\mathbf{X Y Z}}^{\prime}=[1,1,1]^{\mathrm{t}}$, the following results are obtained:

$$
\begin{aligned}
& \mathbf{D}_{2}=\left[\begin{array}{lll}
1 & 0 & 0 \\
0 & 1 & 0 \\
0 & 0 & 1
\end{array}\right] \\
& \mathbf{Z}_{\mathbf{2}}=\left[\begin{array}{cc}
-0.8165 & 0 \\
0.4082 & -0.7071 \\
0.4082 & 0.7071
\end{array}\right], \\
& \mathbf{N}_{\mathbf{2}}=\left[\begin{array}{ccc}
-0.2105 & -0.6047 & 0.4459 \\
0.1666 & -0.1394 & 0.7763
\end{array}\right] \\
& \mathbf{E}_{\mathbf{2}}=\left[\begin{array}{ccc}
0.1719 & -0.2037 & 0.0319 \\
0.4937 & -0.1483 & -0.3454 \\
-0.3640 & -0.3669 & 0.7309
\end{array}\right] \\
& \mathbf{M}_{\mathbf{2}}=\left[\begin{array}{ccc}
1.1719 & -0.2037 & 0.0319 \\
0.4937 & 0.8517 & -0.3454 \\
-0.3640 & -0.3669 & 1.7309
\end{array}\right]
\end{aligned}
$$

The achromatic scale will now be reproduced correctly, with minimum colorimetric error, at the expense of increasing the reproduction error in other regions of the color space since $\mathbf{T}_{\mathbf{R G B}}$ are not strictly color matching functions.

The third option $\mathbf{M}_{3}$ uses the previously known spectral content of the scene, i.e., the spectral reflectances of the ColorChecker chart of the second experiment. This basic colorimetric profile is obtained from Eq. 6 as follows:

$$
\begin{aligned}
& \mathbf{M}_{3}=\mathbf{T}_{\mathbf{X Y Z}}{ }^{\mathrm{t}} \cdot \mathbf{K} \cdot \mathbf{T}_{\mathbf{R G B}} \cdot\left(\mathbf{T}_{\mathbf{R G B}}{ }^{\mathrm{t}} \cdot \mathbf{K} \cdot \mathbf{T}_{\mathbf{R G B}}\right)^{-1} \\
& =\left[\begin{array}{ccc}
1.7478 & 0.3040 & 0.3273 \\
0.9347 & 1.7830 & -0.0171 \\
-0.0384 & 0.1903 & 1.9888
\end{array}\right]
\end{aligned}
$$

Since the autocorrelation matrix $\mathbf{K}$ was designed above with the spectral reflectances $\mathbf{S}$ of the color chart's Munsell chips, we assumed that the illuminant linked to this colorimetric profile was the equal-energy $\mathbf{E}$, and none of those actually used in the second experiment (INC, HWL, DAY). Nevertheless, the colorimetric errors should be smaller than they were in the previous colorimetric profiles. Below, we will determine the degree to which this improvement is significant.

The last basic colorimetric profile $\mathbf{M}_{4}$ belongs to the principal components algorithm group. ${ }^{23}$ It belongs to the a-priori knowledge type because it also uses set $\mathbf{S}$ of the ColorChecker chart as a training set. If the dimensions of $\mathbf{S}$ are $n_{\lambda} \times n$, and $\bar{\rho}$ is the average by rows of the spectral reflectances, then $\bar{s}$ is the average matrix with $n$ repetitions of $\bar{\rho}$. The diagonalization of covariance matrix $\mathbf{K}_{\mathbf{S}}=[\mathbf{S}-\bar{s}] \cdot[\mathbf{S}-\bar{s}]^{\mathrm{t}}$ of the set $\mathbf{S}$ allows us to compute matrix $\mathbf{B}$ from the three eigenvectors associated with the highest first three eigenvalues. Thus, the color transform using principal components for any color-stimulus $\mathbf{C}$ is as follows:

$$
\begin{aligned}
& \hat{\mathbf{t}}_{\mathbf{X Y Z}}^{\prime}=\mathbf{T}_{\mathbf{X Y Z}}{ }^{\mathrm{t}} \cdot \mathbf{B} \cdot\left(\mathbf{T}_{\mathbf{R G B}}{ }^{\mathrm{t}} \cdot \mathbf{B}\right)^{-1} \\
& \left(\mathbf{t}_{\mathbf{R G B}}^{\prime}-\mathbf{T}_{\mathbf{R G B}}{ }^{\mathrm{t}} \cdot \overline{\boldsymbol{\rho}}\right)+\mathbf{T}_{\mathbf{X Y Z}}{ }^{\mathrm{t}} \cdot \overline{\boldsymbol{\rho}}
\end{aligned}
$$

Therefore, in this fourth case, the basic colorimetric profile $\mathbf{M}_{4}$ is:

$$
\begin{aligned}
\hat{\mathbf{t}}_{\mathbf{X Y Z}}^{\prime} & =\mathbf{M}_{\mathbf{4}}\left(\mathbf{t}_{\mathbf{R G B}}^{\prime}-\left[\begin{array}{l}
1.3806 \\
0.9578 \\
1.2404
\end{array}\right]\right)+\left[\begin{array}{l}
3.1144 \\
2.9731 \\
2.5840
\end{array}\right] \\
& =\left[\begin{array}{ccc}
1.7487 & 0.2954 & 0.3264 \\
0.9187 & 1.8215 & -0.0282 \\
-0.0230 & 0.1843 & 2.0002
\end{array}\right] \mathbf{t}_{\mathbf{R G B}}^{\prime}+\left[\begin{array}{c}
-0.0123 \\
0.0050 \\
0.0419
\end{array}\right]
\end{aligned}
$$

Comparing the different matrixes element by element, the first, third and fourth of the basic colorimetric profiles are a priori the most similar. It remains to be seen which of these four best reproduces the colors of any scene.

\section{Theoretical Estimations of the Color Reproduction Level}

The quality factors or figures of merit proposed in the literature for image capture devices are taken as the first options of calculation of the DSC color analysis accuracy index. Basically, without exhaustively analyzing the similarities and differences among these figures of merit, there are two possible approaches: to take into account only the knowledge of the $\mathbf{T}_{\mathbf{X Y Z}}$ and $\mathbf{T}_{\mathbf{R G B}}$ color matching functions without considering the basic colorimetric profile $\mathbf{M}$; and to use another approach to differentiate the quality of the obtained basic colorimetric profiles $\mathbf{M}$. 
This first approach ${ }^{43-45}$ follows the guidelines of the Neugebauer quality factor $q_{N}$ and involves calculating the distance or common volume between the vector subspaces associated with the $\mathbf{T}_{\mathbf{X Y Z}}$ and $\mathbf{T}_{\mathbf{R G B}}$ color matching functions. Keeping the same mathematical notation, the Neugebauer quality factor $q_{N}$ is obtained from the average of the following individual quality factors:

$$
\begin{aligned}
& q_{R}=\frac{\left\|\mathbf{P}_{\mathbf{X Y Z}} \cdot \overline{\mathbf{r}}\right\|^{2}}{\|\overline{\mathbf{r}}\|^{2}}, \quad \text { for red channel } \overline{\mathbf{r}} \\
& q_{G}=\frac{\left\|\mathbf{P}_{\mathbf{X Y Z}} \cdot \overline{\mathbf{g}}\right\|^{2}}{\|\overline{\mathbf{g}}\|^{2}}, \quad \text { for green channel } \overline{\mathbf{g}} \\
& q_{B}=\frac{\left\|\mathbf{P}_{\mathbf{X Y Z}} \cdot \overline{\mathbf{b}}\right\|^{2}}{\|\overline{\mathbf{b}}\|^{2}}, \quad \text { for blue channel } \overline{\mathbf{b}} \\
& \Rightarrow q_{N}=\text { average }\left(q_{R}, q_{G}, q_{B}\right)
\end{aligned}
$$

where $\|\mathbf{x}\|^{2}=\mathbf{x}^{\mathrm{t}} \cdot \mathbf{x}$ is the Euclidean norm and $\mathbf{P}_{\mathbf{X Y Z}}$ is the projector operator of the human visual system characterized by $\mathbf{T}_{\mathbf{X Y Z}}$, i.e., $\mathbf{P}_{\mathbf{X Y Z}}=\mathbf{T}_{\mathbf{X Y Z}}\left(\mathbf{T}_{\mathbf{X Y Z}}{ }^{\mathrm{t}} \cdot \mathbf{T}_{\mathbf{X Y Z}}\right)^{-1} \mathbf{T}_{\mathbf{X Y Z}}{ }^{\mathrm{t}}=$ $\mathbf{O}_{\mathbf{X Y Z}} \cdot \mathbf{O}_{\mathbf{X Y Z}}{ }^{t}$, where $\mathbf{O}_{\mathbf{X Y Z}}$ is the orthonormal color space with color matching functions $\mathbf{T}_{\mathbf{X Y Z}}$ :

$$
\mathbf{O}_{\mathbf{X Y Z}}=\mathbf{T}_{\mathbf{X Y Z}} \cdot\left[\begin{array}{ccc}
-0.1209 & 0.1420 & 0.5715 \\
-0.1019 & 0.1850 & -0.5219 \\
-0.1931 & -0.1865 & -0.0823
\end{array}\right]=\mathbf{T}_{\mathbf{X Y Z}} \cdot \mathbf{M}_{\mathbf{O}}{ }^{\mathrm{t}}
$$

The second option is the Vora quality factor $q_{V}$, which proposes to estimate the fraction of the human vector sub-space spanned within the vector subspace of the image capture device, and is described by:

$$
q_{V}=\frac{\operatorname{tr}\left(\mathbf{P}_{\mathbf{X Y Z}} \cdot \mathbf{P}_{\mathbf{R G B}}\right)}{3}
$$

where $\operatorname{tr}(\mathbf{A})$ is the trace operator of a matrix $\mathbf{A}$ and $\mathbf{P}_{\mathbf{R G B}}$ $=\mathbf{T}_{\mathbf{R G B}}\left(\mathbf{T}_{\mathbf{R G B}}{ }^{\mathrm{t}} \cdot \mathbf{T}_{\mathbf{R G B}}\right)^{-1} \mathbf{T}_{\mathbf{R G B}}{ }^{\mathrm{t}}$ is the projector operator associated with the vector sub-space composed by the color matching pseudo-functions $\mathbf{T}_{\mathbf{R G B}}$.

Reversing the roles of $\mathbf{T}_{\mathbf{X Y Z}}$ and $\mathbf{T}_{\mathbf{R G B}}$, we can calculate a new figure of merit, the color quality factor $C Q F$, defined as follows:

$$
\begin{aligned}
& q_{X}=\frac{\left\|\mathbf{P}_{\mathbf{R G B}} \cdot \overline{\mathbf{x}}\right\|^{2}}{\|\overline{\mathbf{x}}\|^{2}} \\
& q_{Y}=\frac{\left\|\mathbf{P}_{\mathbf{R G B}} \cdot \overline{\mathbf{y}}\right\|^{2}}{\|\overline{\mathbf{y}}\|^{2}} \\
& q_{Z}=\frac{\left\|\mathbf{P}_{\mathbf{R G B}} \cdot \overline{\mathbf{z}}\right\|^{2}}{\|\overline{\mathbf{z}}\|^{2}} \\
& \Rightarrow C Q F=\min \left\{q_{X}, q_{Y}, q_{Z}\right\}
\end{aligned}
$$

Table II shows these figures of color merit for our digital image capture device. If any of the quality factors equals unity, the $\mathbf{T}_{\mathbf{R G B}}$ should be an exact linear combination of $\mathbf{T}_{\mathbf{X Y Z}}$, i.e., a perfect color reproduction should
TABLE II. Color Quality Factors of Our Digital Image Capture Device

\begin{tabular}{clc}
\hline Neugebauer's factor & $\begin{array}{l}q_{R}=0.9342 \\
q_{G}=0.9140 \\
q_{B}=0.9348\end{array}$ & $q_{N}=0.9227$ \\
\hline \multicolumn{2}{c}{$q_{V}=0.9258$} & \\
\hline Vora's factor & \multicolumn{1}{c}{ Color quality factor } & $q_{X}=0.9465$ \\
& $q_{Y}=0.9465$ \\
$q_{z}=0.9361$ & \\
\hline
\end{tabular}

be achieved. It is clear that our digital image capture device does not fulfil the Luther condition and, therefore, there will be systematic color errors in raw performance. One of the purposes of this work will be to determine and quantify these color errors and to find simple procedures for compensating them.

The second approach ${ }^{46-48}$ focuses on minimizing the mean-squared error metric in CIE-XYZ or its orthonormal $\mathbf{O}_{\mathbf{X Y Z}}$ or CIE-L*a*b* spaces, so we can determine which of the four options of basic colorimetric profiles $\mathbf{M}$ performs best. The mathematical formalism is as follows:

- If the figure of merit is based on the mean-squared error metric in CIE-1931 XYZ color space, it is defined by:

$$
q_{I}\left(\mathbf{T}_{\mathbf{X Y Z}}, \mathbf{T}_{\mathbf{R G B}}\right)=\frac{\operatorname{tr}\left(\mathbf{M} \cdot \mathbf{T}_{\mathbf{R G B}}^{\mathrm{t}} \cdot \mathbf{T}_{\mathbf{X Y Z}}\right)}{\operatorname{tr}\left(\mathbf{T}_{\mathbf{R G B}}{ }^{\mathrm{t}} \cdot \mathbf{T}_{\mathbf{X Y Z}}\right)}
$$

where in the third and fourth options $\mathbf{T}_{\mathbf{R G B}}$ is replaced by $\mathbf{K} \cdot \mathbf{T}_{\mathbf{R G B}}$.

- If the figure of merit is based on the mean-squared error metric in the orthonormal space $\mathbf{O}_{\mathbf{x y z}}$ derived from the CIE-1931 XYZ color space, it is defined by:

$$
q_{O}\left(\mathbf{T}_{\mathbf{X Y Z}}, \mathbf{T}_{\mathbf{R G B}}\right)=\frac{\operatorname{tr}\left(\mathbf{O}_{\mathbf{X Y Z}} \cdot \mathbf{M}_{\mathbf{O}} \cdot \mathbf{M} \cdot \mathbf{T}_{\mathbf{R G B}}{ }^{\mathrm{t}}\right)}{\operatorname{tr}\left(\mathbf{P r}_{\mathbf{X Y Z}}\right)}
$$

with the same replacements as above.

- Finally, if the figure of merit is based on the meansquared error metric CIE-L*a*b* uniform color space, it is defined by:

$$
\begin{aligned}
& q_{a p}\left(\mathbf{T}_{\mathbf{X Y Z}}, \mathbf{T}_{\mathbf{R G B}}\right)=\frac{\operatorname{tr}\left(\mathbf{F}_{\mathbf{l a b}}{ }^{\mathrm{t}} \cdot \mathbf{F}_{\mathbf{l a b}} \cdot \mathbf{M} \cdot \mathbf{T}_{\mathbf{R G B}}{ }^{\mathrm{t}} \cdot \mathbf{T}_{\mathbf{X Y Z}}\right)}{\operatorname{tr}\left(\mathbf{F}_{\mathbf{l a b}}{ }^{\mathrm{t}} \cdot \mathbf{F}_{\mathbf{l a b}} \cdot \mathbf{T}_{\mathbf{R G B}}{ }^{\mathrm{t}} \cdot \mathbf{T}_{\mathbf{X Y Z}}\right)} \\
& \mathbf{F}_{\mathbf{l a b}}=\frac{1}{3}\left[\begin{array}{ccc}
0 & 116 & 0 \\
500 & -500 & 0 \\
0 & 200 & -200
\end{array}\right]
\end{aligned}
$$

with the same replacements as above.

With this formalism we can see in Table III that basic colorimetric profile no. 2 is qualitatively the best, but we do not know whether the differences observed between the four profiles would really be significant when treated as psychoquantitative color differences, i.e., using a realistic color appearance model. Therefore, 
TABLE III. Color Quality Factors Obtained by Minimizing the Mean-Squared Error Metric for the Four Basic Colorimetric Profiles of our Digital Image Capture Device.

\begin{tabular}{|c|c|c|c|c|}
\hline Color space & M1 & M2 & M3 & M4 \\
\hline CIE-1931 XYZ & $q_{1}=2.0772$ & $q_{1}=1.4524$ & $q_{1}=2.1205$ & $q_{1}=2.3339$ \\
\hline Orthonormal CIE-XYZ & $q_{0}=0.9258$ & $q_{0}=0.7334$ & $q_{0}=0.9995$ & $q_{0}=0.9989$ \\
\hline CIE $-L^{*} a^{*} b^{*}$ & $q_{a p}=1.4369$ & $q_{a p}=1.3640$ & $q_{1}=2.0931$ & $q_{a p}=1.9340$ \\
\hline
\end{tabular}

we think that it is advisable to find new alternatives to these theoretical DSC color analysis accuracy indexes. We propose one alternative in the following section using a CIE-L*a*b* color space, which is the simplest way to describe the luminous capture of a digital image capture device and to compare this device dependent color encoding with the human visual system. Other alternative procedures using complex color appearance models, which operate with psychoquantitative data, will be the subject of other study.

\section{Ideal Gray Balance}

A gray balance algorithm is used initially to obtain the relative colorimetric $R G B$ values from the digital RGB output levels. If $N D O L_{k}$ is the normalized digital output level for each color-channel, the relative RGB values are obtained from the ratio between the areas beneath the color-matching functions associated with the camera. Because this ratio is not $1: 1: 1$, the gray balance is applied as follows:

$$
\begin{aligned}
& N D O L_{k}=\frac{D O L_{k}}{2^{\text {bits }}-1}, \quad k=R, G, B \\
& R=\frac{N D O L_{R}}{b a l_{R}} ; \quad G=\frac{N D O L_{G}}{b a l_{G}} ; \quad B=\frac{N D O L_{B}}{b a l_{B}}
\end{aligned}
$$

Therefore, a RGB triplet equal to $(0.2,0.2,0.2)$ will be associated directly with a spectrally neutral color or any equivalent metamer for the device, even when their digital RGB encodings are originally very different.

Going back to Eq. 2, we now have both color relative specifications as they relate to the same white reference, i.e., the equal-energy stimulus $\mathbf{E}$. Therefore, the above operation is crucial when one is attempting to convert a digital still camera into a tele-colorimeter. If we consider the existence of digital dark noise $f_{k}$ in the image sensor, the gray balance algorithm is described as Eq. 24.

Reproduction Model with Luminance Adaptation

In the second stage, a colorimetric profile is proposed with luminance adaptation as a function of the control of the f-number $N$. This algorithm adapts the relative and shorter dynamic range of the capture device response, limited by the spectral dynamic range of the $O E C S F s$, to the real dynamic luminance range of any scene. For instance, let us consider a gray scale test pattern (Fig. 5, center) well characterized by its XYZ tristimulus values (in $\mathrm{cd} / \mathrm{m}^{2}$ ) using a telespectroradiometer. This pattern is captured with certain $\left(N_{0}, t_{0}=20 \mathrm{~ms}\right)$ values but the darker and lighter grays are clipped by dark noise and by saturation of the image sensor respectively. If the lens aperture $N$ decreases to $N_{1}$, the exposure level will rise, so the RGB digital output levels will now be different (Fig. 5, top). However, the absolute tristimulus values XYZ (in cd/ $\mathrm{m}^{2}$ ) obtained by a tele-spectroradiometer will remain fixed. Now, the lighter grays will again be clipped at the expense of optimally encoding the darker grays, which were not clipped by dark noise. If the lens aperture $N$ increases to $N_{2}$, the exposure level will decrease again, so the RGB digital output levels will again be different (Fig. 5, bottom), although the absolute tristimulus values XYZ will remain fixed. The darker grays will again be clipped at the expense of optimally encoding the lighter grays, which were not clipped by saturation. Then, the key issue is how to compensate the variation of the lens aperture $N$ in order to always obtain the same CIE-XYZ values. The same issue applies to the variation of the photosite integration time $t$, but in this work we will focus only on the faster change of exposure $H$ with f-number $N$ (Eq. 7).

Because our purpose in this work is to get an absolute estimation, in $\mathrm{cd} / \mathrm{m}^{2}$, of the tristimulus values $\mathrm{XYZ}$, we

$$
\begin{gathered}
\mathbf{t}_{\mathbf{R G B}}^{\prime}=\left[\begin{array}{l}
R \\
G \\
B
\end{array}\right]=\mathbf{B a l} \cdot\left[\begin{array}{l}
D O L_{R}-f_{R} \\
D O L_{G}-f_{G} \\
D O L_{B}-f_{B}
\end{array}\right], \\
\operatorname{where~Bal}=\left[\begin{array}{ccc}
\frac{1}{b a l_{R}\left[\left(2^{b i t s}-1\right)-f_{R}\right]} & 0 & 0 \\
0 & \frac{1}{b a l_{G}\left[\left(2^{b i t s}-1\right)-f_{G}\right]} & \frac{1}{b a l_{B}\left[\left(2^{b i t s}-1\right)-f_{B}\right]} \\
0 & 0
\end{array}\right.
\end{gathered}
$$

$$
\Rightarrow \quad \hat{\mathbf{t}}_{\mathbf{X Y Z}}^{\prime}=\mathbf{M} \cdot \mathbf{t}_{\mathbf{R G B}}^{\prime} \quad \text { with } \quad \mathbf{T}_{\mathbf{X Y Z}}{ }^{\mathrm{t}}=\mathbf{M} \cdot \mathbf{T}_{\mathbf{R G B}}{ }^{\mathrm{t}}
$$




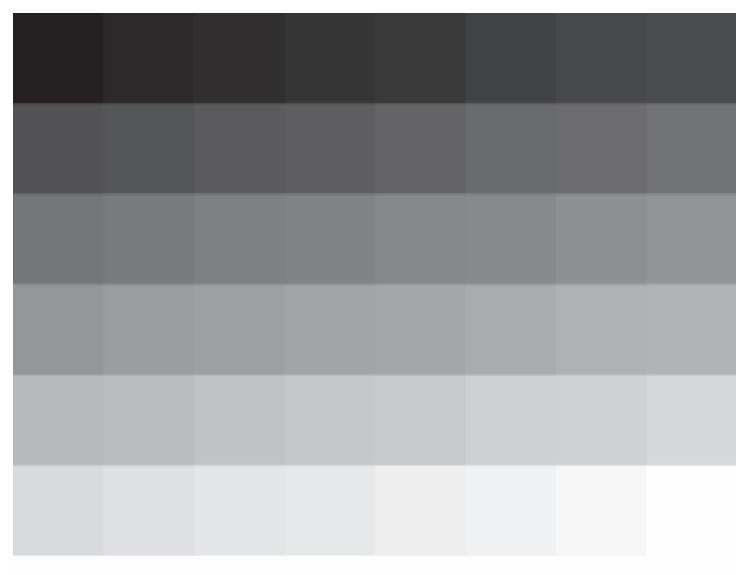

must link the group of luminances of the former gray scale test pattern with the corresponding RGB data of our color device. This relationship was already introduced in the camera opto-electronic conversion function (OECF). For any exposure determination-varying the lens aperture $N$ or photosite integration time $t$-the $O E C F s$ are described by Eq. 2, but they must be reversed in order to be inserted correctly into the colorimetric profile:

$$
\begin{aligned}
& \forall(N, t) \text { exposure determination: } \\
& \mathbf{t}_{\mathbf{R G B}}^{\prime}=\left[\begin{array}{l}
R \\
G \\
B
\end{array}\right]=\left[\begin{array}{l}
O E C F_{R}(L) \\
O E C F_{G}(L) \\
O E C F_{B}(L)
\end{array}\right] \\
& \rightarrow \hat{\mathbf{t}}_{\mathbf{X Y Z}}^{\prime}=\frac{1}{L_{\mathrm{E}}} \mathbf{M}\left[\begin{array}{l}
O E C F_{R}^{-1}(R) \\
O E C F_{G}^{-1}(G) \\
O E C F_{B}^{-1}(B)
\end{array}\right]
\end{aligned}
$$
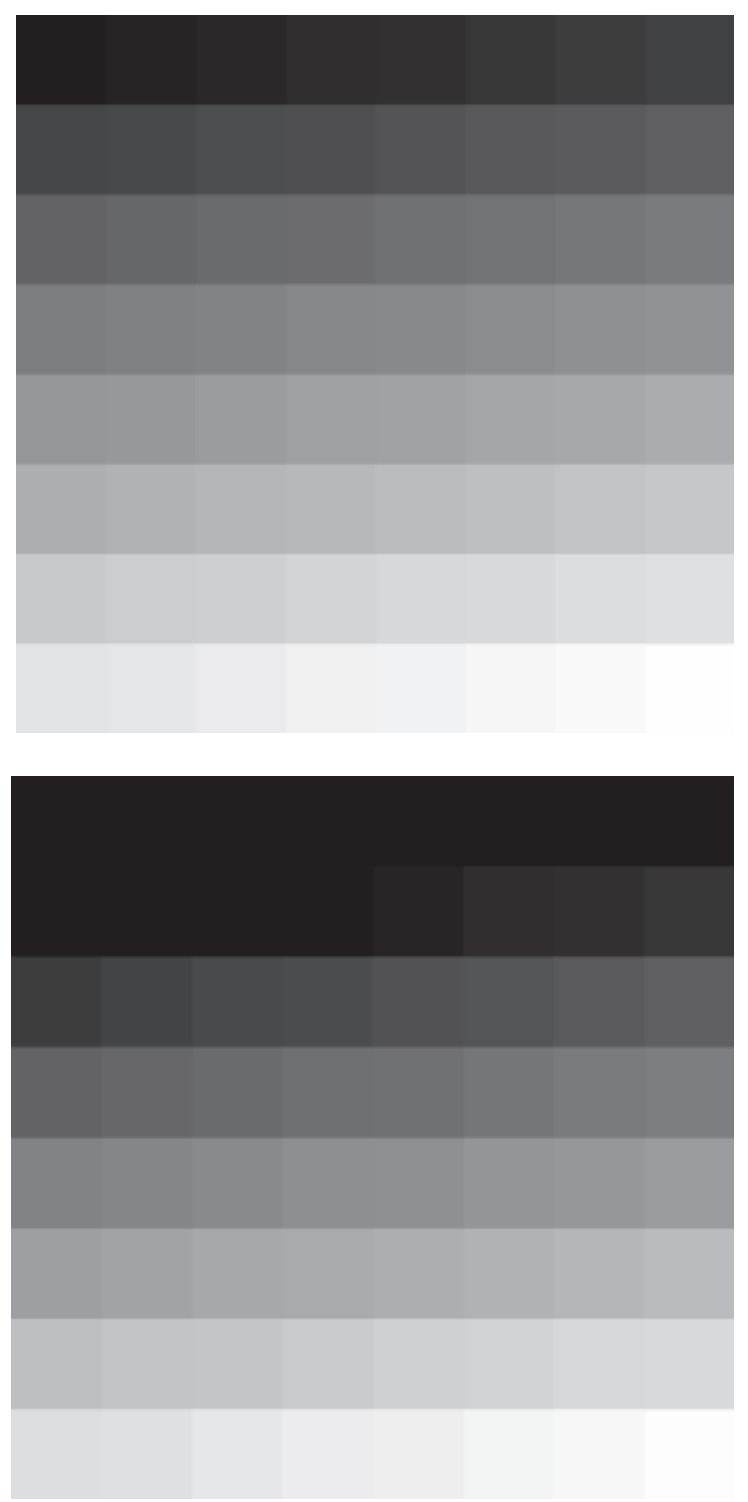

where $L$ is the luminance of the gray patch and $L_{\mathrm{E}}$ is the luminance of the perfect white diffuser in the gray scale test pattern.

Unlike the ISO 14524 standard, ${ }^{21}$ which proposes an empirical method for obtaining the $O E C F s$ using a gray scale test pattern but not necessarily the equal-energy illuminant $\mathbf{E}$, we propose an algorithm for obtaining the $O E C F s$ from the OECSFs using a simulated spectrally neutral gray scale test pattern illuminated by equalenergy $\mathbf{E}$. This procedure is equivalent to obtaining the responses of our color device to a large set of equal-energy stimuli $\mathbf{E}$ with variable uniform exposure levels $H_{\mathrm{E}}$ (with uniform radiance $L_{e}$ and total luminance $L_{\mathrm{E}}$ ). The total luminance $L_{\mathrm{E}}$ of each spectrally neutral gray patch is easily computed from its corresponding uniform radiance $L_{e}$. By setting the photosite integration time $t$ $=t_{0}=20 \mathrm{~ms}$ and giving free the lens aperture $N$, we can estimate the relative colorimetric RGB values for each gray patch or, equivalently, for each possible luminance $L_{\mathrm{E}}$ of the adapted white:

$$
\begin{aligned}
& L_{e}=\frac{L_{E}}{K_{m}\left(\overline{\mathbf{y}}^{\mathrm{t} \cdot \mathbf{E}}\right) \Delta \lambda}=\frac{L_{E}}{683 \cdot 21.3714 \cdot 5} \\
& \frac{N D O L_{k}}{b a l_{k}}=\frac{1}{b a l_{k}} \sum_{380 \mathrm{~nm}}^{780 \mathrm{~nm}} \frac{L_{e}}{N^{2}} t_{0} r_{k}\left(\lambda, \frac{L_{e}}{N^{2}} t_{0}\right) \Delta \lambda \\
& \frac{N D O L_{k}}{b a l_{k}}=\frac{1}{b a l_{k}} \sum_{380 \mathrm{~nm}}^{780 \mathrm{~nm}}\left[\begin{array}{l}
a_{\lambda k}+\frac{b_{\lambda k}}{1+\exp \left(-\frac{L_{e}}{N^{2}} t_{0}-c_{\lambda k}\right.} \\
d_{\lambda k}
\end{array}\right] \Delta \lambda
\end{aligned}
$$

Figure 6 shows the balance of luminance adaptation of our digital image capture device, where each triad of $O E C F s$ curves belongs to a ( $N$, fixed $t)$ scale exposure series where, from left to right, $N=\{1,1.4,2,2.8,4,5.6$, $8,11,16,22.4\}$. It can be seen clearly that the $O E C F s$ curves are parallel. This is characteristic of any adaptation process, such as, for instance, the luminance adaptation described by any color appearance model. ${ }^{63,64}$ 


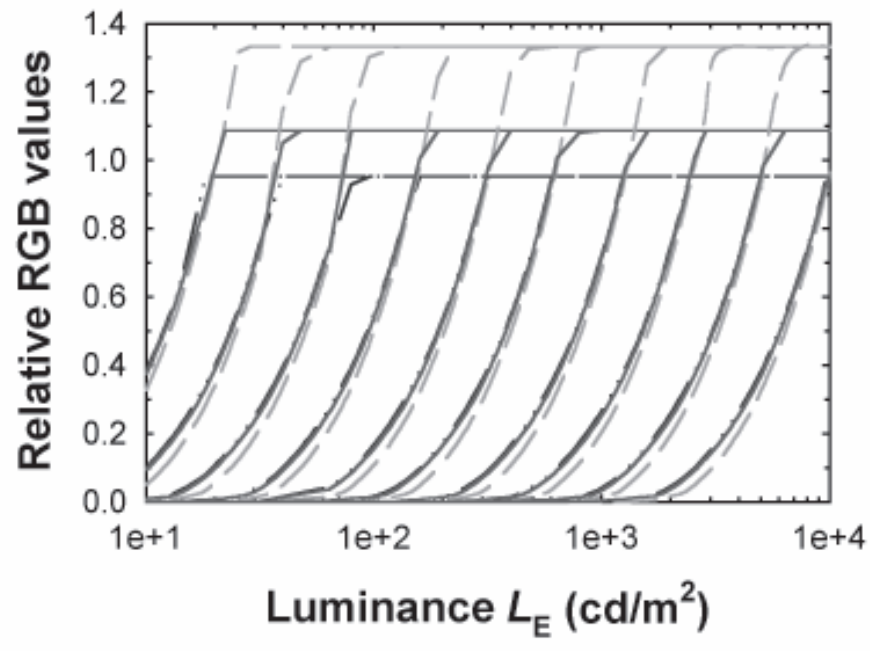

F'igure 6. Balance of luminance adaptation of our digital image capture device. Each triad of OECFs curves belongs to a $(N$, fixed $t$ ) scale exposure series. From left to right, $N=1,1.4$, $2,2.8,4,5.6,8,11.2,16,22.4\}$. Solid line: red channel; dashed line: green channel; dash-dot-dot: blue channel.

For example, a luminance of $100 \mathrm{~cd} / \mathrm{m}^{2}$ is encoded better with $N=2.8$ than with $N=4$. On the other hand, a luminance of $1000 \mathrm{~cd} / \mathrm{m}^{2}$ is encoded optimally with both $N=8$ (larger relative RGB values) and $N=11$ (smaller relative $\mathrm{RGB}$ values). However, if the rest of luminances of the scene are higher than this value, $N=11$ is better than $N=8$. If the rest of the luminances are lower, the opposite is true. Therefore, although the ISO DSC dynamic range of our color device is short (10:1 for the three color channels), we have found an algorithm to compensate for this handicap, which is typical in the majority of digital image capture devices.

The obtained OECFs show a linear behavior in the mid-range when the $\mathrm{x}$-axis (luminance) is not logarithmic, so the inverse $O E C F s$ can be considered as straight lines in this range except for the two tails associated with under-exposure and saturation. If we amplify the central zone of the last graph (Fig. 7), two additional examples may be provided to help us understand the balance of luminance adaptation that will be inserted in the reproduction model (Eq. 25) of our color device. A spectrally neutral gray with luminance $150 \mathrm{~cd} / \mathrm{m}^{2}$ gives almost saturated responses in the three color channels with $N=2.8$. With $N=4$, it would be captured better. However, with $N=5.6$ the signal is closer to the encoding noise level. Alternatively, with f-numbers $N=5.6,4$ and 2.8, we obtain the same relative colorimetric RGB encoding (0.4) for three grays of luminances 300, 160 and $75 \mathrm{~cd} / \mathrm{m}^{2}$. The key issue is how we can compensate the variation of the lens aperture $N$ to obtain the corresponding CIE-XYZ values.

Therefore, the linear inverse OECFs can be modeled with a slope $m$ and an offset value $h$ for each color channel as follows:

$$
\begin{aligned}
& O E C F_{R}^{-1}(R)=m_{R}(N) \cdot R+h_{R}(N) \\
& O E C F_{G}{ }^{-1}(G)=m_{G}(N) \cdot G+h_{G}(N) \\
& O E C F_{B}{ }^{-1}(B)=m_{B}(N) \cdot B+h_{B}(N)
\end{aligned}
$$

Fitting the $m$ versus $N$ and $h$ versus $N$ data (Fig. 8), we have found that these relationships are second-poly-

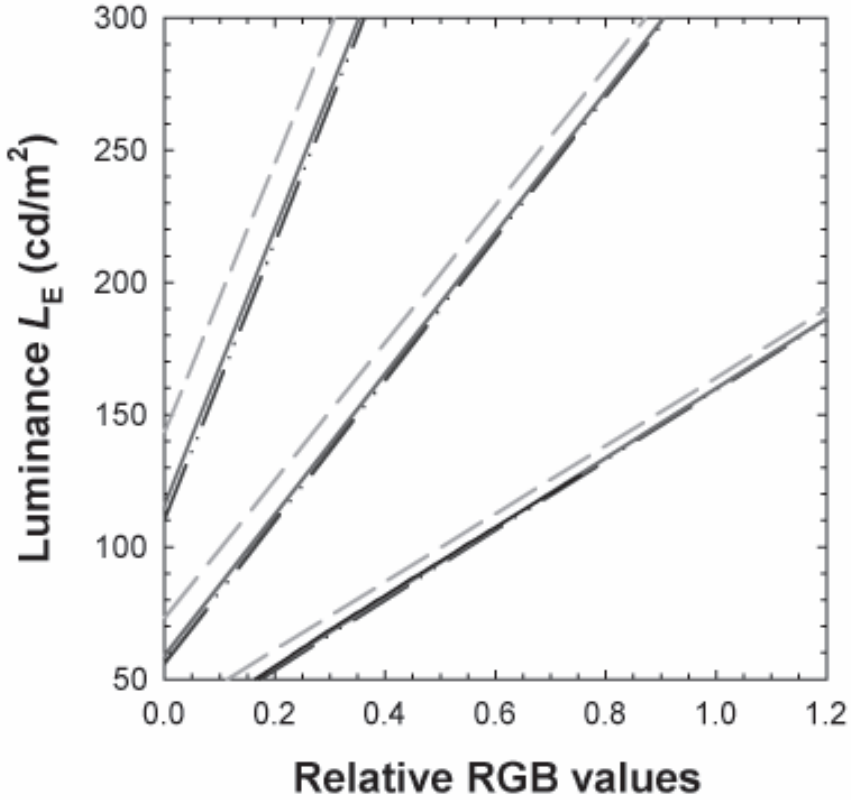

Figure 7. Linear representation of the inverse OECFs of Fig. 10 for the values $N=5.6,4$, and 2.8. Solid line: red channel; dashed line: green channel; dash-dot-dot: blue channel.

nomial (Eq. 28), and their fitting parameters are shown in Table IV:

$$
\begin{aligned}
& m_{k}(N)=m_{0 k}+m_{1 k} N+m_{2 k} N^{2} \\
& h_{k}(N)=h_{0 k}+h_{1 k} N+h_{2 k} N^{2}
\end{aligned}
$$

From our point of view, the similarity of these parameters in the three color channels, and particularly of those associated with the slope $m$, justify the balance of luminance adaptation being an inherent property of the image sensor. If any optoelectronic parameter is changed, the OECSFs will change and, therefore, so will the OECFs. Furthermore, the light variability in the offset value $h$ might be associated with the color architecture of our color device.

Thus, we can now describe the colorimetric profile with luminance adaptation for any digital still camera as follows, using absolute tristimulus values directly, without taking into account the luminance $L_{\mathrm{E}}$ of the adapted white:

\section{Original - referred image data}

(from a tele-spectroradiometer):

$\mathbf{t}_{\mathbf{X Y Z}}=\left[\begin{array}{c}X \\ Y \\ Z\end{array}\right] \frac{\mathrm{cd}}{\mathrm{m}^{2}}$

Scene-referred image data (from a characterized DSC):

$\hat{\mathbf{t}}_{\mathbf{X Y Z}}=$

$\left[\begin{array}{c}\hat{X} \\ \hat{Y} \\ \hat{Z}\end{array}\right]=\mathbf{M}\left\{\left[\begin{array}{ccc}m_{R}(N) & 0 & 0 \\ 0 & m_{G}(N) & 0 \\ 0 & 0 & m_{B}(N)\end{array}\right] \cdot\left[\begin{array}{c}R \\ G \\ B\end{array}\right]+\left[\begin{array}{c}h_{R}(N) \\ h_{G}(N) \\ h_{B}(N)\end{array}\right]\right\} \frac{\mathrm{cd}}{\mathrm{m}^{2}}$ 

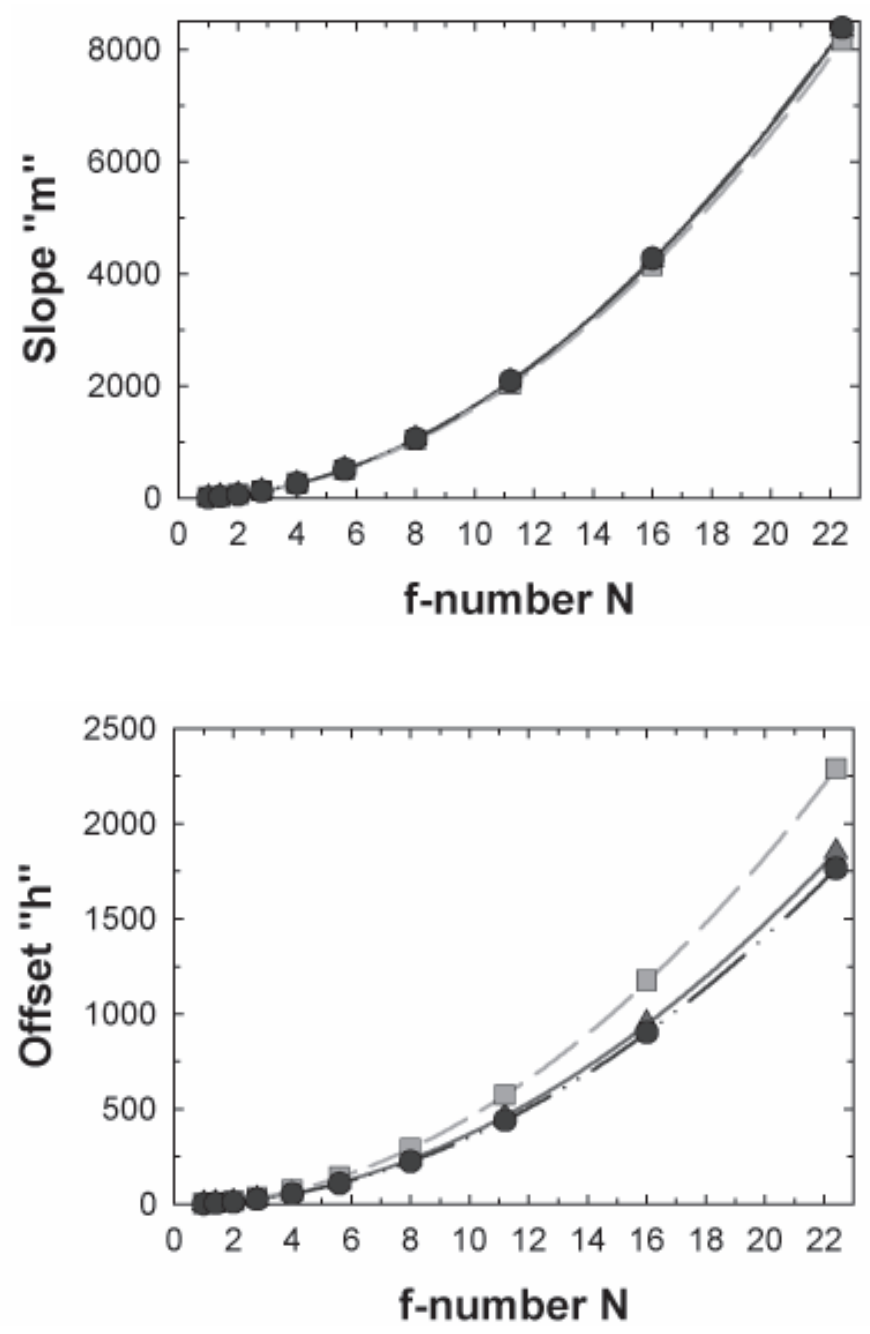

Figure 8. Top: Second polynomial fit of the slope " $m$ " of the linear inverse OECFs versus $f$-number $N$ of the zoom lens (circles: B channel; squares: $\mathrm{G}$ channel; trianges: $\mathrm{R}$ channel). Bottom: Second polynomial fit of the offset " $h$ " of the linear inverse OECFs versus $f$-number $N$ of the zoom lens (circles: B channel; squares: G channel; trianges: $\mathrm{R}$ channel).

where $\mathbf{M}$ is the basic colorimetric profile with four possible options as explained above and the RGB values are the gray-balanced digital output levels $D O L_{k}$ (Eq. 24).

\section{Linear Model of Color Correction}

As mentioned above, we will use the viewing booth experiment with the ColorChecker chart under three light sources to test the reproduction model with luminance adaptation ( $N=4$ for the INC lamp, $N=5.6$ for the HWL and DAY lamps). Therefore, the first step of this stage of the colorimetric characterization is to compare the estimated absolute tristimulus values XYZ, from the model associated with the Sony DXC-930P 3CCD-RGB camera, with the measured tristimulus values XYZ from the Photo Research PR-650 telespectroradiometer. This comparison, plotted in Figs. 9 through 11 for the maximum ignorance basic colorimetric profile $\mathbf{M}_{1}$, indicates that a color correction is necessary to better predict the scene colorimetry. A linear model of color correction, as proposed in Eq. 30, is the
TABLE IV. Fitting Parameters of the Second Order Polynomial of the Slope and Offset Value of the Inverse OECF for Each Color Channel as a Function of the Lens Aperture $\mathbf{N}$.

\begin{tabular}{ccccccc}
\hline & \multicolumn{3}{c}{ Slope "m" } & \multicolumn{3}{c}{ Offset value "h" } \\
Channel & $m_{0}$ & $m_{1}$ & $m_{2}$ & $h_{0}$ & $h_{1}$ & $h_{2}$ \\
\hline $\mathrm{R}$ & 2.9328 & -1.1753 & 16.6933 & -1.8589 & 0.7488 & 3.6676 \\
$\mathrm{G}$ & 4.2475 & -1.7476 & 16.3244 & -2.5022 & 1.0540 & 4.5253 \\
$\mathrm{~B}$ & 2.1729 & -0.7980 & 16.7543 & -1.3288 & 0.4843 & 3.5035 \\
\hline
\end{tabular}

TABLE V. Fitting Parameters of the Linear Color Correction of the Reproduction Model According to the Basic Colorimetric Profile

\begin{tabular}{ccccccc}
\hline & \multicolumn{3}{c}{ Offset value "a" } & \multicolumn{3}{c}{ Slope "b" } \\
& $a_{X}$ & $a_{y}$ & $a_{z}$ & $b_{X}$ & $b_{y}$ & $b_{z}$ \\
\hline $\mathrm{M}_{1}$ & -32.65 & -35.02 & -30.10 & 0.1851 & 0.1577 & 0.1937 \\
$\mathrm{M}_{2}$ & -15.40 & -23.77 & 12.81 & 0.3314 & 0.3384 & 0.2748 \\
$\mathrm{M}_{3}$ & -31.15 & -33.65 & -29.63 & 0.1773 & 0.1549 & 0.1952 \\
$\mathrm{M}_{4}$ & -30.79 & -33.54 & -31.11 & 0.1785 & 0.1538 & 0.1893 \\
\hline
\end{tabular}

most simple option among the nonlinear methods available, and seems to work well (Figs. 9-11). This is understandable since our color device will show systematic reproduction errors because its $\mathbf{T}_{\mathbf{R G B}}$ color matching functions do not fulfil the Luther condition:

$\mathbf{t}_{\mathbf{X Y Z}}=\left[\begin{array}{c}X \\ Y \\ Z\end{array}\right]=\mathbf{A}_{\mathbf{C}}+\mathbf{B}_{\mathbf{C}} \cdot \hat{\mathbf{t}}_{\mathbf{X Y Z}}=\left[\begin{array}{c}a_{X} \\ a_{Y} \\ a_{Z}\end{array}\right]+\left[\begin{array}{ccc}b_{X} & 0 & 0 \\ 0 & b_{Y} & 0 \\ 0 & 0 & b_{Z}\end{array}\right] \cdot\left[\begin{array}{c}\hat{X} \\ \hat{Y} \\ \hat{Z}\end{array}\right]$

where $\mathbf{A}_{\mathbf{C}}$ is the offset tristimulus vector and $\mathbf{B}_{\mathbf{C}}$ is the scaling diagonal matrix. These parameters are shown in Table $\mathrm{V}$ for the four possible basic colorimetric profiles $\mathbf{M}$.

However, with this linear color correction model we can analyze all the spectral and colorimetric characterization proposed until now in perspective. The steps that we must reconfigure are the following:

- To definitively scale the color matching pseudo-functions with the diagonal matrix $\mathbf{B}_{\mathbf{C}}$, i.e., to substitute $\mathbf{T}_{\mathbf{R G B}}$ for $\mathbf{T}_{\mathbf{R G B}} \cdot \mathbf{B}_{\mathbf{C}}$ where it is necessary. The reason for this reconfiguration is that the former $\mathbf{T}_{\mathbf{R G B}}$ depended on the value $b a l_{B}=1$ of the white balance test, so a multiplicative factor is missed for each color channel (XYZ) in the proposed characterization model (Eq. 30). This multiplicative factor is $\mathbf{B}_{\mathbf{C}}$, obtained by comparing the estimated XYZ data with the measured XYZ data (Figs. 9-11). Therefore, this means that in general the true or absolute scaling of $\mathbf{T}_{\mathbf{R G B}}$ relative to $\mathbf{T}_{\mathbf{X Y Z}}$ is decreased by approximately $80 \%$ in the maximum ignorance case $\left(b_{X}, b_{Y}, b_{Z} \cong 0.2\right)$, so our digital image capture device needs more light (almost double: two steps fewer in the f-number $N$ ) than the standard observer CIE-1931 XYZ in order to offer the same chromatic results qualitatively. Finally, Fig. 12 shows the color matching functions $\mathbf{T}_{\mathbf{R G B}}$ (with the multiplicative factor $\mathbf{B}_{\mathbf{C}}$ included) versus $\mathbf{T}_{\mathbf{X Y Z}}$ with the absolute scaling of $\mathbf{T}_{\mathbf{X Y Z}}$.

- To define the raw colorimetric profile (with luminance adaptation) as the former reproduction model scaled by diagonal matrix $\mathbf{B}_{\mathbf{C}}$, and to set apart the 


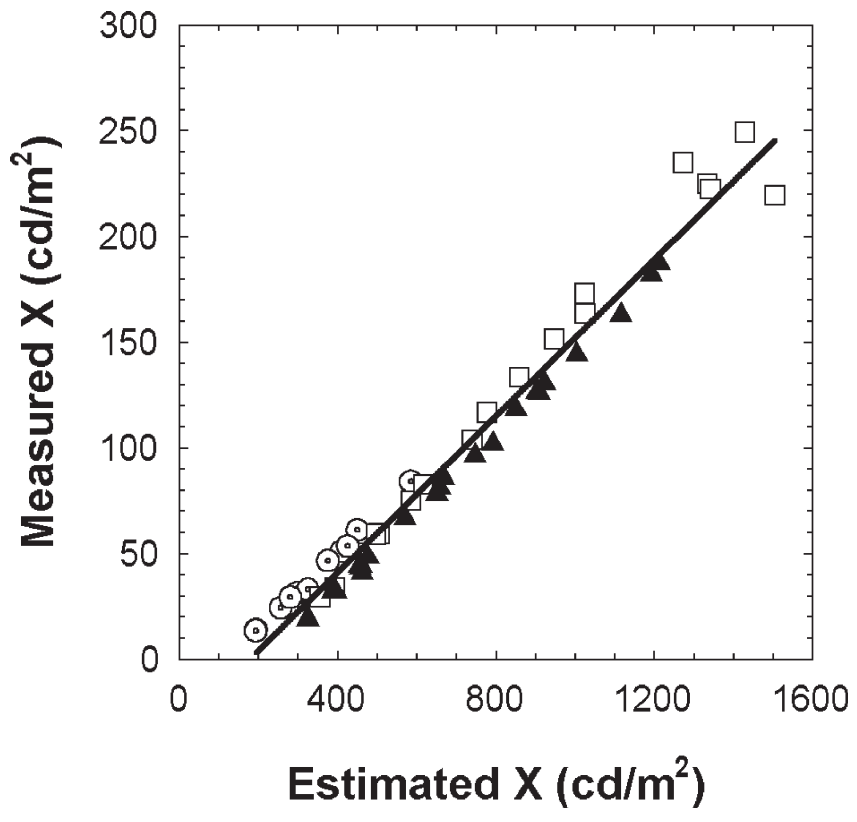

Figure 9. Linear correction (solid line) of the tristimulus value $X$ of the color data using the raw reproduction model with luminance adaptation and basic colorimetric profile MaxIgLS (correlation index $r^{2}=0.9761$ ). (Dotted circles: samples under incandescent lamp; squares: samples under metal halide lamp; triangles: samples under daylight fluorescent lamp.)

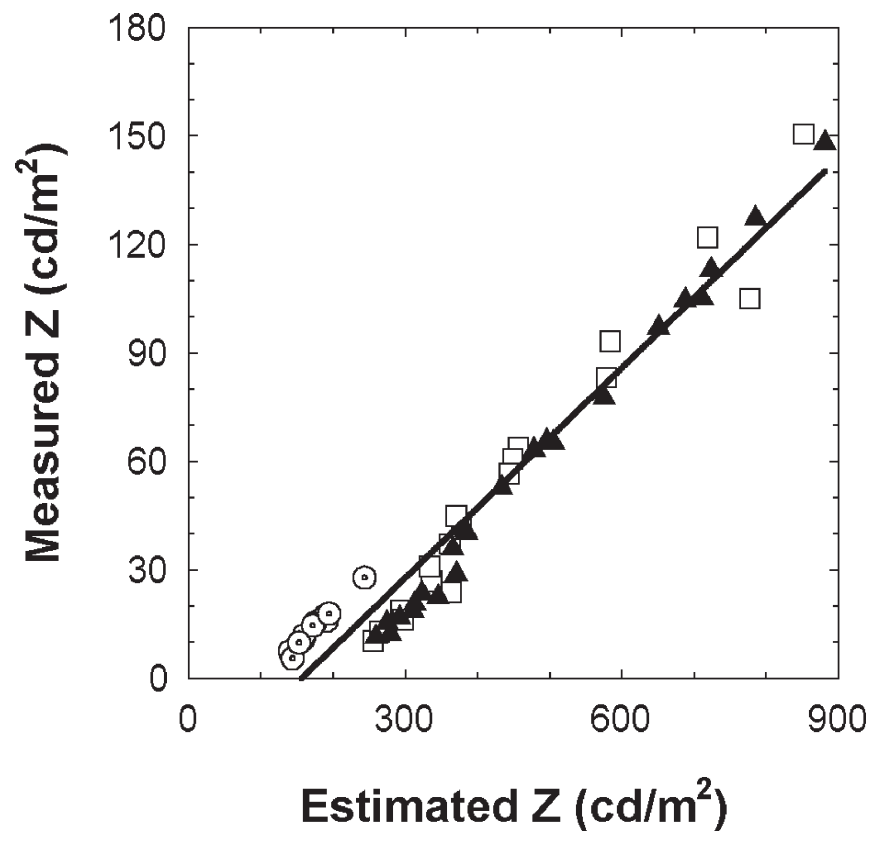

Figure 11. Linear correction (solid line) of the tristimulus value $Z$ of the color data using the raw reproduction model with luminance adaptation and basic colorimetric profiel MaxIgLS (correlation index $r^{2}=0.9512$ ). (Dotted circles: samples under incandescent lamp; squares: samples under metal halide lamp; triangles: samples under daylight fluorescent lamp.)

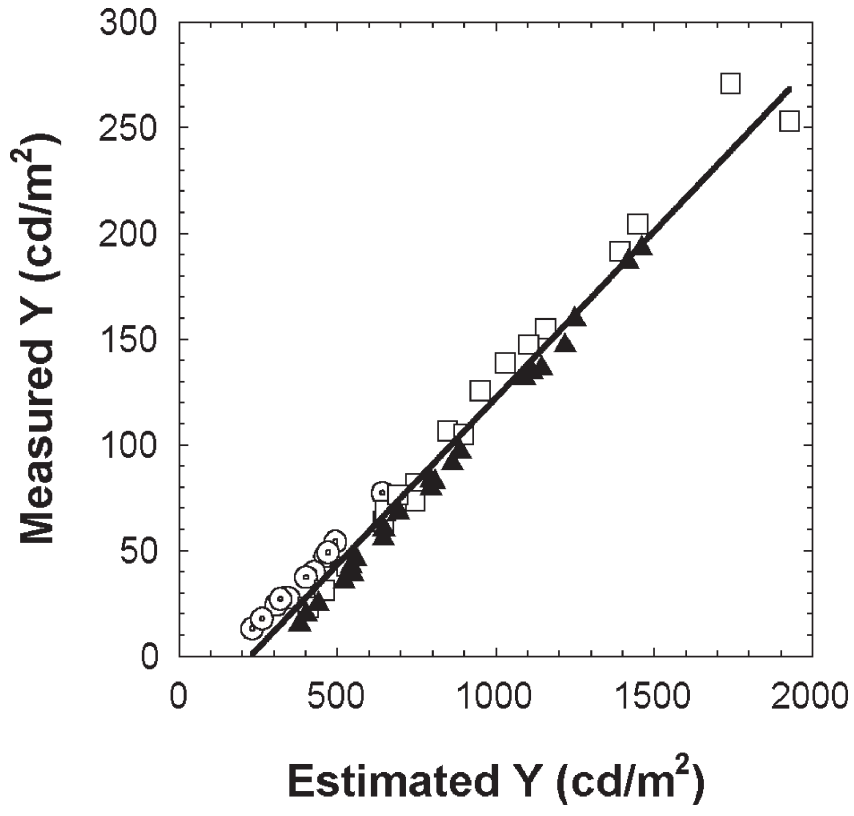

Figure 10. Linear correction (solid line) of the tristimulus value $Y$ of the color data using the raw reproduction model with luminance adaptation and basic colorimetric profile MaxIgLS (correlation index $r^{2}=0.9761$ ). (Dotted circles: samples under incandescent lamp; squares: samples under metal halide lamp; triangles: samples under daylight fluorescent lamp.)

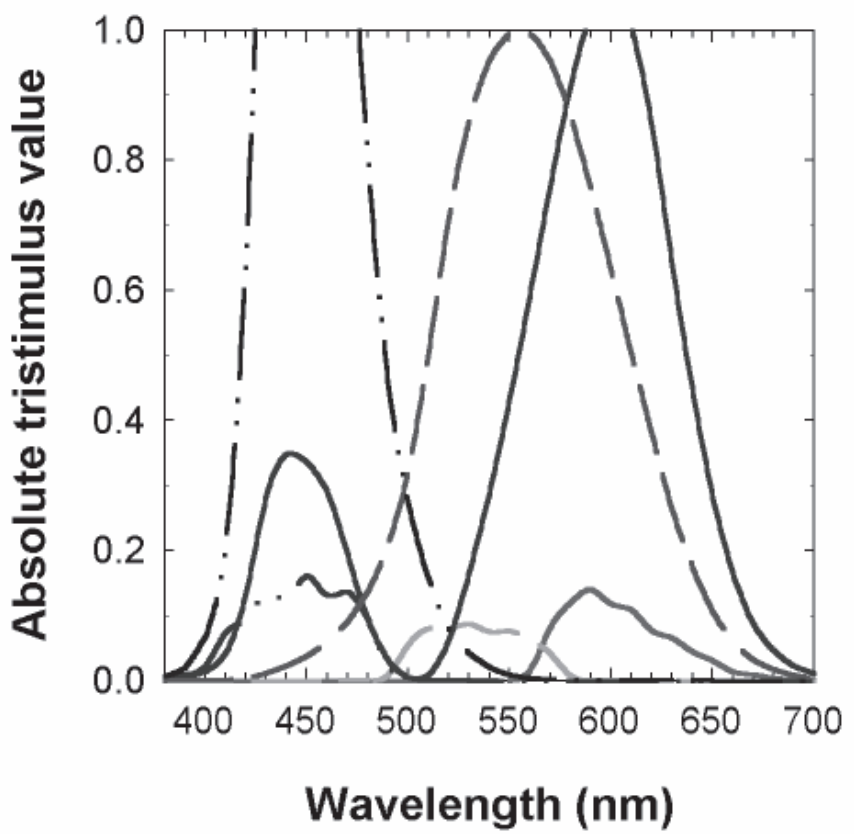

Figure 12. Absolute scaling of the color-matching pseudofunctions of our digital image capture device as they relate to Standard Observer CIE-1931 XYZ (solid line: R or X channel; dashed line: G or Y channel; dash-dot-dot line: B or Z channel). 
offset tristimulus vector $\mathbf{A}_{\mathbf{C}}$. This vector is the only parameter describing the systematic color deviations of our color device, which means it is the error-associated bias with the difference between true and estimated values, determined using the calibrated DSC and a tele-spectroradiometer respectively. Therefore, since we always use raw RGB data from the camera to avoid the influence of any uncontrolled post-processing, the tristimulus vector $\mathbf{A}_{\mathbf{C}}$ should really be the only variable of the color correction model justified by the technological impositions on the design of the spectral sensitivities (mismatch of the color matching functions) of the digital image capture device.

\section{DSC Color Accuracy Analysis Index}

In our final reproduction model with luminance adaptation, there are two well-differentiated colorimetric profiles: raw (without $\mathbf{A}_{\mathbf{C}}$ ) and compensated (with $\mathbf{A}_{\mathbf{C}}$ ), with which any DSC scene analysis error metrics can be calculated. At first approximation, we will use the CIELAB color space to obtain the DSC scene analysis error metrics, i.e., we will analyze the supposed beneficial effect of the correction vector $\mathbf{A}_{\mathbf{C}}$ by means of the CIE-L*a*b* pseudo-uniform color space. All the graphical results will refer to the reproduction model with the maximum ignorance case, with the basic colorimetric profile $\mathbf{M}_{1}$. Afterwards, a comparison of the four options for basic colorimetric profiles will be carried out in order to establish which of them best predicts the scene colorimetry.

Although the capture of each color patch of the ColorChecker chart under three lamps was clearly isolated, the CIE-L*a*b* transform may adapt itself to an isolated mode using the corresponding luminance $L_{\mathrm{E}}$ of the adapted white (Tables A1-A3 in the Appendixavailable as Supplemental Material). Figure 13 shows in $\left(\mathrm{C}^{*}, \mathrm{~L}^{*}\right)$ and $\left(\mathrm{a}^{*}, \mathrm{~b}^{*}\right)$ profiles the estimated data (hollow circles) associated with the raw colorimetric profile (with $\mathbf{M}_{1}$ and without $\mathbf{A}_{\mathbf{C}}$ ) relative to the measured data (solid circles). It can be seen in the $\left(a^{*}, b^{*}\right)$ profile that the estimated data are more centered than the measured data, which indicates that our color device systematically reproduces in the raw state a smaller chroma: that it desaturates the colors. This behavior also appears in the $\left(\mathrm{C}^{*}, \mathrm{~L}^{*}\right)$ profile because the estimated data are grouped towards the achromatic axis. On the other hand, in the $\left(\mathrm{C}^{*}, \mathrm{~L}^{*}\right)$ profile, it can also be seen that the hollow symbols plot at a higher lightness $\mathrm{L}^{*}$ than the solid symbols, which indicates that our color device systematically reproduces in the raw state a higher lightness: that it lightens the colors. The estimation of the hue is affected less than the other perceptual variables, see Table VI. Taking into account the correction vector $\mathbf{A}_{\mathbf{C}}$, the chromatic results improve (Fig. 14), but not sufficiently to pass any pass/fail industrial test (see Table VI), because the average of the total color difference $\Delta \mathrm{E}_{94}$ is higher than 6 and is clearly perceptible, in all basic colorimetric profile options.

This final chromatic result using the CIELAB algorithm should not be considered highly unsatisfactory, since the initial conditions imposed to test our color reproduction model are very demanding. To begin with, the training set is completely spectral (OECSFs and $\mathbf{T}_{\mathbf{R G B}}$ ) and different from the test set, which is the ColorChecker chart under three real light sources (not CIE illuminants) inside a non-standard light box. Moreover, neither the spectral characteristics of the light sources nor the patches of the color chart are used. In
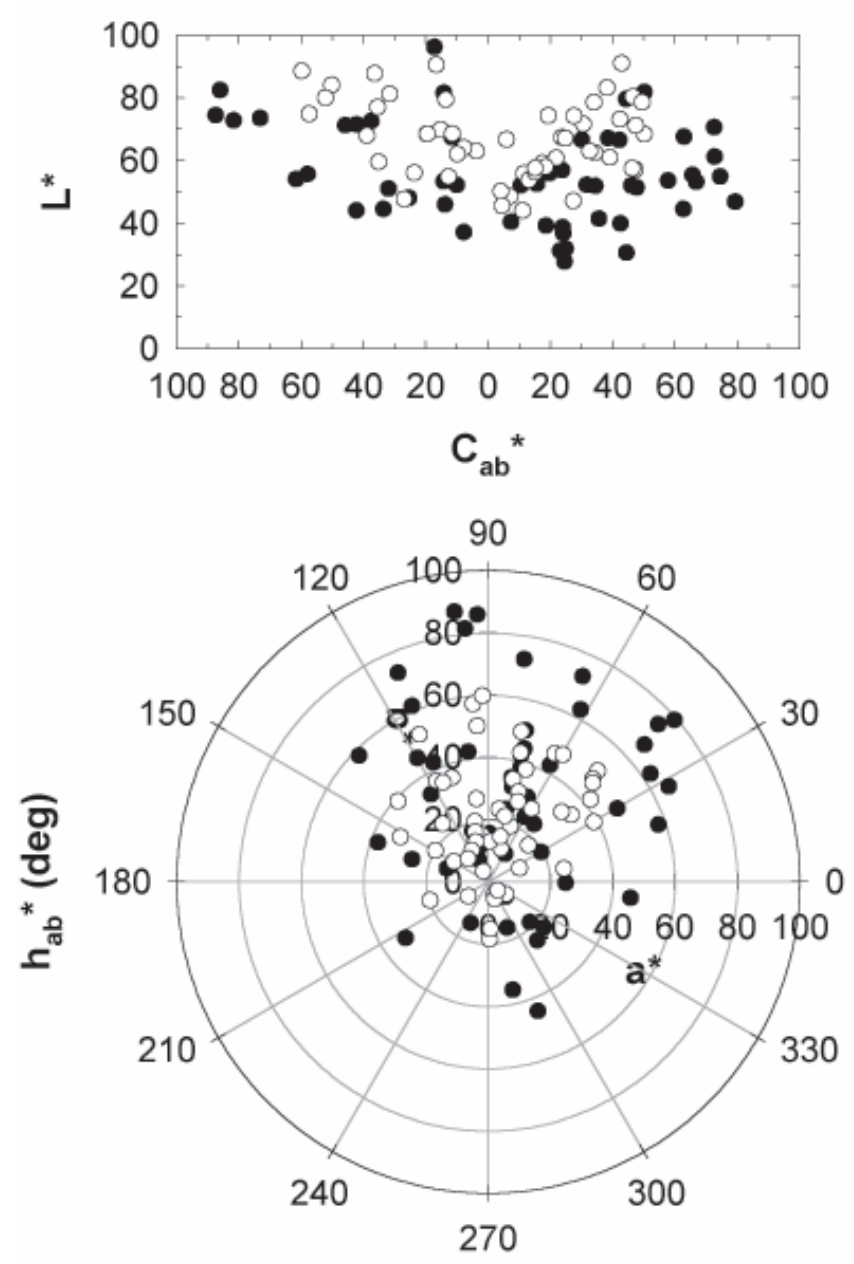

Figure 13. Comparison of the color data estimated with the raw reproduction model (hollow circles) and color data (solid circles) measured using the CIE- $\left(a^{*}, b^{*}\right)$ and $\mathrm{CIE}-\left(\mathrm{C}^{*}, \mathrm{~L}^{*}\right)$ profiles.

Hunt's terminology, ${ }^{65}$ we are trying to reach exact color reproduction for our digital image capture device, but the quality of the attained color reproduction is good, as can be seen from Color Plate 5, p. 374. The raw and compensated estimated data and the measured data were encoded in sRGB format ${ }^{11}$ to visually simulate the colorimetric estimation of the scene. The color charts on the left are the predictions of the raw colorimetric profile (with $\mathbf{M}_{1}$ ) under each lamp (from top to bottom); the central color charts are the measured data from the tele-spectroradiometer; and the color charts on the right are the predictions with the compensated colorimetric profile. It is clear that, without individual color corrections for any particular color, the general valuation of the reproduced ColorChecker chart under the daylight fluorescent lamp is excellent, and even more so if this color reproduction is visualized when the original chart is not present, which is the conventional visualization mode of softcopy and hardcopy images.

Because of the colorimetric imperfections in the design of the spectral sensitivities due to technological conditions, the raw color reproduction of our color device is not perfect, as the colors are systematically encoded lighter and more desaturated. This camera behavior can 

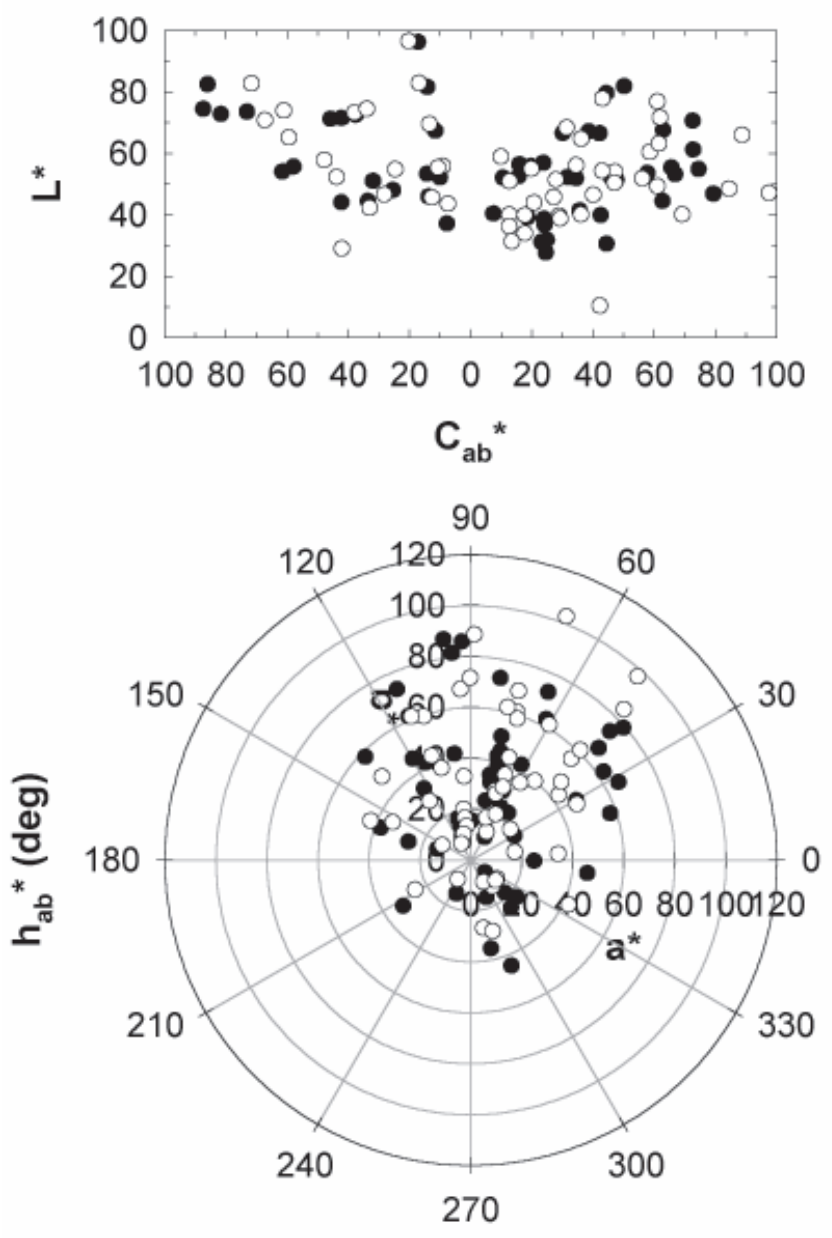

Figure 14. Comparison of the color data estimated with the corrected reproduction model (open circles) and color data (solid circles) measured using the $\mathrm{CIE}-\left(\mathrm{a}^{*}, \mathrm{~b}^{*}\right)$ and $\mathrm{CIE}-\left(\mathrm{C}^{*}, \mathrm{~L}^{*}\right)$ profiles.

be due to the mismatch of the color matching functions of the camera, and is very similar to the effect of the Ives-Abney-Yule compromise, ${ }^{66,67}$ in relation to the design of the spectral sensitivities of an image capture device. Since the spectral sensitivities are all positive, although the Luther condition is not fulfilled, this arrangement mainly confines the color errors to excesses of lightness and losses of chroma (Table VI). However, unlike the Ives-Abney-Yule compromise, which uses a non linear correction factor, ${ }^{66}$ a tristimulus vector $\mathbf{A}_{\mathbf{C}}$ as a linear correction model is enough to yield a good level of exact color reproduction (which corrects the lightness better than the chroma).

Although the proposed color reproduction model with luminance adaptation is partially inexact, we think that this can be improved by increasing the number of color channels, for instance, from three (RGB) to six (RGB and R'G'B'). This can be done by placing a color filter before the camera or even by working with a monochrome camera with $n$ color filters (multispectral imaging). In any event, from this new perspective, we think that there are enough research lines to follow in the coming years. These might include the comparison of the raw color reproduction levels of various commercial cameras.
Comparison of the Basic Colorimetric Profiles

Until now, all the numerical and graphical results were associated with the color reproduction model with the maximum ignorance case $\left(\mathbf{M}_{1}\right)$. With the above tools we can attempt to find which of the four possible basic colorimetric profile best predicts the scene colorimetry. The numerical data of Table VI in CIELAB format show that the worst is basic profile no. 2 (MaxIgWPPLS), contradicting the results derived from the color quality factors (Tables II and III). On the other hand, basic profile no. 1 (MaxIgLS) reaches the same results as basic profiles no. 3 (LSAPK) and no. 4 (PC) in spite of the minimal knowledge assumptions applied to the scene. Therefore, from our point of view, it is redundant to elucidate between the minimal and maximal knowledge assumptions of the scene. It is more important to find new methods for the analysis of the raw reproduction level of the digital image capture devices and to improve the accuracy of the color correction model applied to them.

\section{Conclusions}

A new color characterization model with luminance adaptation for digital still cameras has been proposed. This reproduction model, separated into two spectral and colorimetric characterization parts, would allow the use of any digital image capture device-both cameras and scanners, although for scanners, the f-number $N$ must be kept fixed-as a photometer and colorimeter. This means that, as in photometers and colorimeters, it is not necessary to know in advance, the spectral content of a scene for this new color instrument to perform correctly.

The performance of the color device must be separated into raw and compensated. Due to the engineering and technological conditions of the design of the spectral sensitivities of these color devices, the raw color reproduction undergoes certain systematic color deviations that are very hard to detect: the colors of any scene will be encoded lighter and more desaturated in the raw performance or state. Obviously, in these conditions, our color device cannot perform like a tele-colorimeter. However, with just a tristimulus vector $\left(\mathbf{A}_{\mathbf{C}}\right)$ as a color correction model, the scene colorimetry estimation improves significantly. Although the reproduction level obtained is not completely exact, if we take the compensated scene-referred image data without comparing them with the original-referred image data, the color appearance of the estimated or reproduced image is reasonably good, as can be seen in Color Plate 5, p. 374.

Although this new reproduction model enables the user to choose between some basic colorimetric profiles according to the previous knowledge of the spectral content of the scene, we have found that the option with maximum ignorance by least squares regression performs as well as other basic colorimetric profiles, which have a given spectral content. Therefore, this analysis highlights the versatility of a digital image capture device as a photometer and colorimeter.

The proposed color characterization algorithm can be used as an analysis tool to compare commercial cameras, particularly regarding their raw performance or state. However, the major applications of a colorimetrically characterized digital camera as an absolute telecolorimeter belong in industrial colorimetry. If we add to this the ability of simultaneously measuring the whole visual field, unlike true tele-spectroradiometers, this new color instrument is suitable for performing the color characterization of displays, microscopic and/or non- 
Table VI: Average Absolute Color Deviations in CIE-L*a*b* Space of the Raw and Corrected Reproduction Model According to the Basic Colorimetric Profile. (Profile no. 1: MaxlgLS, no. 2: MaxIgWPPLS; no. 3: LSAPK; no. 4: PC.)

\begin{tabular}{|c|c|c|c|c|c|c|c|}
\hline No. & $\left|\Delta \mathrm{L}^{*}\right|$ & $\left|\Delta a^{*}\right|$ & $\left|\Delta b^{*}\right|$ & $\left|\Delta C^{*}\right|$ & $\left|\Delta \mathrm{H}^{\star}\right|$ & $\Delta \mathrm{E}$ & $\Delta \mathrm{E}_{94}$ \\
\hline 1 (raw) & 11.75 & 7.80 & 10.24 & 13.32 & 3.41 & 19.70 & 13.14 \\
\hline 1 (corr.) & 4.32 & 6.00 & 8.80 & 10.37 & 3.87 & 12.71 & 6.73 \\
\hline 2 (raw) & 8.66 & 12.44 & 27.99 & 27.37 & 12.93 & 34.25 & 17.88 \\
\hline 2 (corr.) & 2.72 & 11.73 & 15.81 & 16.16 & 10.48 & 22.12 & 11.21 \\
\hline 3 (raw) & 11.41 & 6.10 & 10.16 & 11.75 & 4.25 & 18.52 & 12.78 \\
\hline 3 (corr.) & 4.11 & 6.06 & 8.91 & 10.05 & 3.88 & 12.56 & 6.58 \\
\hline 4 (raw) & 11.39 & 6.13 & 10.81 & 12.19 & 4.73 & 19.03 & 12.93 \\
\hline 4 (corr.) & 4.12 & 6.40 & 10.01 & 10.97 & 4.69 & 13.72 & 7.04 \\
\hline
\end{tabular}

homogeneous samples (biochemical processes, marbles and granites, etc.). It is also an alternative solution for understanding and controlling the color appearance of complex images, which is so important in realistic color management flowcharts in digital TV and cinema (camera and display interconnection).

Acknowledgment. This research was supported by the Comisión Interministerial de Ciencia y Tecnología (CICYT) (Spain) under grant TAP99-0856 and by the Ministerio de Ciencia y Tecnología (Spain) under grant DPI2002-00118.

\section{References}

1. ISO/DIS 12231 (2001), Photography - Electronic Still Picture Imaging - Terminology; http://www.pima.net/standards/iso/tc42/wg18/ wg18.htm.

2. ISO/WD 22028-1.16 (2001), Photographic and Graphic Technology Extended Colour Encodings for Digital Image Storage, Manipulation and Interchange - Part 1: Architecture and requirements; http:// www.pima.net/standards/iso/tc42/wg18/ wg18.htm.

3. H. R. Kang, Color Technology for Electronic Imaging Devices, SPIE Press, Bellingham, WA, 1997, p. 177.

4. ISO/FDIS 12234-1 (2001), Photography - Electronic Still Picture Imaging - Removable Memory: Basic Removable-Memory Model; http:/ /www.pima.net/standards/ iso/tc42/wg18/wg18.htm

5. ISO/FDIS 12234-2 (2001), Photography - Electronic Still Picture Imaging - Removable Memory: Image Data Format - TIFF/EP; http:// www.pima.net/standards/ iso/tc42/wg18/wg18.htm.

6. ISO/FDIS 12234-3 (2001), Photography - Electronic Still Picture Imaging - Removable Memory: Design Rule for Camera File System (DCF); http://www.pima.net/ standards/iso/tc42/wg18/wg18.htm.

7. ISO/WD 17321-1 (2001), Graphic Technology and Photography Colour Characterization of Digital Still Cameras (DSCs) - Part 1: Stimuli, Metrology, and Test Procedures; http://www.pima.net/standards/ iso/tc42/WG20/wg20.htm.

8. ISO/WD 17321-2 (2002), Graphic Technology and Photography Colour Characterization of Digital Still Cameras (DSCs) - Part 2: Methods for Determining Transforms from Raw DSC to Scene-Referred Image Data by Minimising Errors in Colour Spaces; http:// www.pima.net/standards/ iso/tc42/WG20/wg20.htm.

9. R. W. G. Hunt, The Reproduction of Colour, $5^{\text {th }}$ ed., Fountain Press, Kingston-upon-Thames, 1995, p. 82.

10. J. Morovic and M. R. Luo, The Fundamentals of Gamut Mapping: A Survey, J. Imaging. Sci. Technol. 45, 283 (2001).

11. IEC 61966-2-1 Ed. 1: 1999, Multimedia Systems and Equipment Colour Measurement and Management - Part 2-1: Colour Management - Default RGB colour space - sRGB; http://www.srgb.com.

12. PIMA WD 7667 (2001), Photography - Electronic Still Picture Imaging - Extended sRGB Color Encoding - e-sRGB; http://www.pima.net/ standards/it10/it10.htm.

13. ICC 1:2001 (version 4), File Format for Color Profiles; http:// www.color.org.

14. ISO 2846-2:2000, Graphic Technology - Colour and Transparency of Ink Sets for Four-Colour Printing - Part 2: Coldset Offset Lithographic printing, ISO, Geneva, 2000; http://www.iso.ch.

15. R. Ramanath, Demosaicking Methods for Bayer Color Arrays, J. Electron. Imaging 11, 306 (2002).

16. H.J. Trussell and R.E. Hartwig, Mathematics for Demosaicking, IEEE Trans. Image Process. 11, 485 (2002).

17. H.J. Trussell, Application of Set Theoretic Methods to Color Systems, Color Res. Appl. 16, 31 (1991).
18. D.H. Brainard, Colorimetry, in Handbook of Optics, vol. I, $2^{\text {nd }}$ ed., Optical Society of America, New York, NY, 1995, chap. 26.

19. G. Sharma and H.J. Trussell, Digital Color Imaging, IEEE Trans. Image Process. 6, 901 (1997).

20. M. J. Vhrel and H.J. Trussell, Color Device Calibration: A Mathematical Formulation, IEEE Trans. Image Process. 8, 1796 (1999).

21. ISO 14524: 1999, Photography - Electronic Still Picture Cameras Methods for Measuring Opto-Electronic Conversion Functions (OECFs), ISO, Geneva, 1999; http://www.iso.ch.

22. G.D. Finlayson and M.S. Drew, Constrained Least Squares Regression in Colour Spaces, J. Electron. Imaging 6, 484 (1997).

23. M.J. Vhrel and H.J. Trussell, Color Correction Using Principal Components, Color. Res. Appl. 14, 328 (1992).

24. J. Holm, I. Tastl and S. Hordley, Evaluation of DSC (Digital Still Camera) Scene Analysis Error Metrics - Part 1, in Proc. IS\& T/SID 8h $^{\text {th }}$ Color Imaging Conf., IS\&T, Springfield, VA 2000, pp. 279-287.

25. P.G. Herzog, Colorimetric Characterization of a Novel Multiple-Channel Sensors for Imaging and Metrology, J. Electronic Imaging 8, 324 (1999).

26. S. Tominaga, Spectral Imaging by a Multichannel Camera, J. Electron. Imaging 8, 332 (1999).

27. M.J. Vhrel and H.J. Trussell, Filter Considerations in Color Correction, IEEE Trans. Image Process. 3, 147 (1994).

28. M.J. Vhrel, H.J. Trussell and J. Bosch, Design and Realization of Optimal Color Filters for Multi-Illuminant Correction, J. Electronic Imaging 4, 6 (1995).

29. P.L. Vora and H.J. Trussell, Mathematical Methods for the Design of Color Scanning Filters, IEEE Trans. Image Process. 6, 312 (1997).

30. S. Quan, Optimal Design of Camera Spectral Sensitivity Functions Based on Practical Filter Components, in Proc. IS\&T/SID gth Color Imaging Conf., IS\&T, Springfield, VA 2000, pp. 326-331.

31. J.Y. Hardeberg, Multispectral Image Acquisition and Simulation of Illuminant Changes, in Colour Imaging: Vision and Technology, L.W. MacDonald and M.R. Luo, Eds., John Wiley and Sons, Chichester, 1999.

32. F.H. Imai, R.S. Berns and D-Y. Tzeng, A Comparative Analysis of Spectral Reflectance Estimated in Various Spaces Using a Trichromatic Camera System, J. Imaging Sci. Technol. 44, 280 (2000).

33. R.S. Berns, The Science of Digitizing Paintings for Color-Accurate Image Archives: A Review, J. Imaging Sci. Technol. 45, 305 (2001).

34. ISO 12641: 1997, Graphic Technology - Prepress Digital Data Exchange - Color Targets for Input Scanner Calibration, ISO, Geneva, 1997; http://www.iso.ch.

35. H. R. Kang, Color Technology for Electronic Imaging Devices, SPIE Press, Bellingham, WA, 1997, p. 67

36. G-W. Chang and Y-C. Chen, Colorimetric Modeling for Vision Systems, J. Electronic Imaging 9, 432 (2000).

37. G. Hong, M.R. Luo and P.A. Rhodes, A Study of Digital Camera Colorimetric Characterization Based on Polynomial Modeling, Color Res. Appl. 26, 76 (2001).

38. W. Wu, J.P. Allebach and M. Analoui, Imaging Colorimetry Using a Digital Camera, J. Imaging Sci. Technol. 44, 267 (2000).

39. R.S. Berns and M.J. Shyu, Colorimetric Characterization of a Desktop Drum Scanner Using a Spectral Model, J. Electronic Imaging 4, 360 (1995).

40. G. Sharma, Targetless Scanner Color Calibration, J. Imaging Sci. Technol. 44, 301 (2000).

41. H. Kotera, Generation of Virtual Spectral Color Target and Application to Testing Input Devices, J. Imaging Sci. Technol. 45, 373 (2001).

42. F. Martínez-Verdú, J. Pujol, P. Capilla, Calculation of the Color Matching Functions of Digital Cameras from Their Complete Spectral Sensitivities, J. Imaging Sci. Technol. 46, 15 (2002).

43. H.E.J. Neugebauer, Quality Factor for Filters Whose Spectral Transmittances Are Different Form Color Mixture Curves, and Its Application to Color Photography, J. Opt. Soc. Amer. 46, 821 (1956). 
44. P.L. Vora and H.J. Trussell, Measure of Goodness of a Set of ColorScanning Filters, J. Opt. Soc. Amer. A 10, 1499 (1993).

45. M. Wolski, Optimization of Sensor Response Functions for Colorimetry of Reflective and Emissive Objects, IEEE Trans. Image Process. 5, 507 (1996).

46. G. Sharma and H.J. Trussell, Figures of Merit for Color Scanners, IEEE Trans. Image Process. 6, 990 (1997).

47. P.L. Vora and H.J. Trussell, Mathematical Methods for the Analysis of Color Scanning Filters, IEEE Trans. Image Process. 6, 321 (1997).

48. G. Sharma, H.J. Trussell and M.J. Vhrel, Optimal Non-Negative Color Scanning Filters, IEEE Trans. Image Process. 7, 129 (1998).

49. S. Quan and N. Ohta, Evaluating Hypothetical Spectral Sentivities with Quality Factors, J. Imaging Sci. Technol. 46, 8 (2002).

50. M.D. Fairchild, Color Appearance Models, Addison-Wesley, New York, 1998.

51. Ref. 9, p. 451.

52. Ref. 9, p. 463.

53. R. Luther, Aus dem Gebiet der Farbreizmetrik, Z. Tech. Phys. 8, 540 (1927).

54. H.E. Ives, The Transformation of Color Mixture Equations From One System To Another, J. Franklin Inst. 16, 673 (1915).

55. B.K.P. Horn, Exact Reproduction of Colored Images, Comput. Vis. Graph. Image Process. 26, 135 (1984).
56. V.C. Cardei and B. Funt, Color Correcting Uncalibrated Digital Images, J. Imaging Sci. Technol. 44, 288 (2000).

57. G.D. Finlayson and P.M. Morovic, Metamer Constrained Color Correction, J. Imaging Sci. Technol. 44, 295 (2000).

58. J. Pujol, F. Martínez-Verdú and P. Capilla, Método Para la Caracterización Espectrocolorimétrica de Dispositivos de Captura. Request code, P2001 02667 (20/11/2001), Spain.

59. J. Pujol, F. Martínez-Verdú and P. Capilla, Dispositivo Para la Medida del Color Basado en Una Cámara CCD-RGB Convencional, Request code: P2001 02668 (20/11/2001), Spain.

60. F. Martínez-Verdú, Spectroradiometric Characterization of the Spectral Linearity of a Conventional Digital Camera, Proc. SPIE3648, 280 (1999).

61. G.C. Holst, CCD Arrays, Cameras and Displays, $2^{\text {nd }}$ ed., SPIE Press, Bellingham, WA, 1998.

62. J.R. Janesick, Scientific Charge-Coupled Devices, SPIE Press, Bellingham, WA, 2001.

63. Ref. 9, p. 714

64. R.W.G. Hunt, Measuring Colour, $3^{\text {rd }}$ ed., Fountain Press, Kingstonupon-Thames, 1998 , p. 239.

65. Ref. 9, p. 222.

66. Refg, 9 p. 128.

67. Ref. 9 , p. 453. 

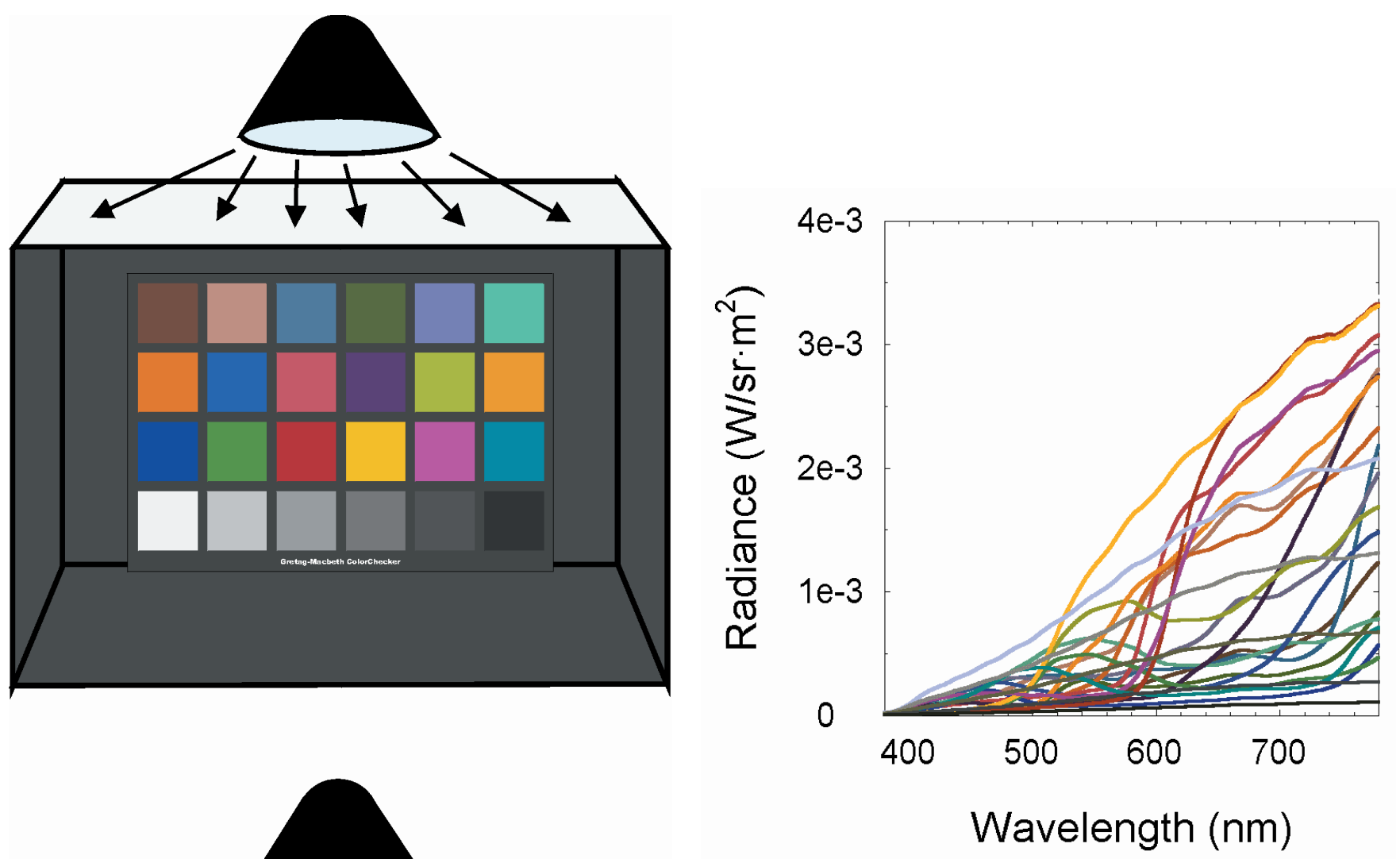

Color Plate 1. ColorChecker chart inside the viewing booth (top: related mode; bottom: isolated mode). (Francisco MartínezVerdú, et al, pp.279-295).

Color Plate 2. Top: Color stimuli of the ColorChecker Chart under incandescent light scource (INC lamp). Bottom: Simulation of the image captured by our digital image capture device. (Francisco Martínez-Verdú, et al, pp.279-295).

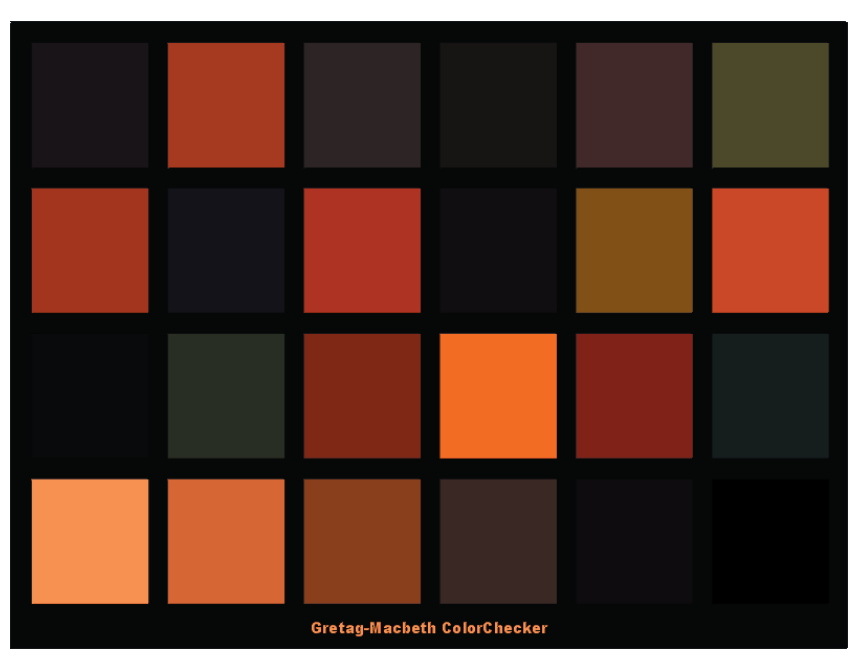



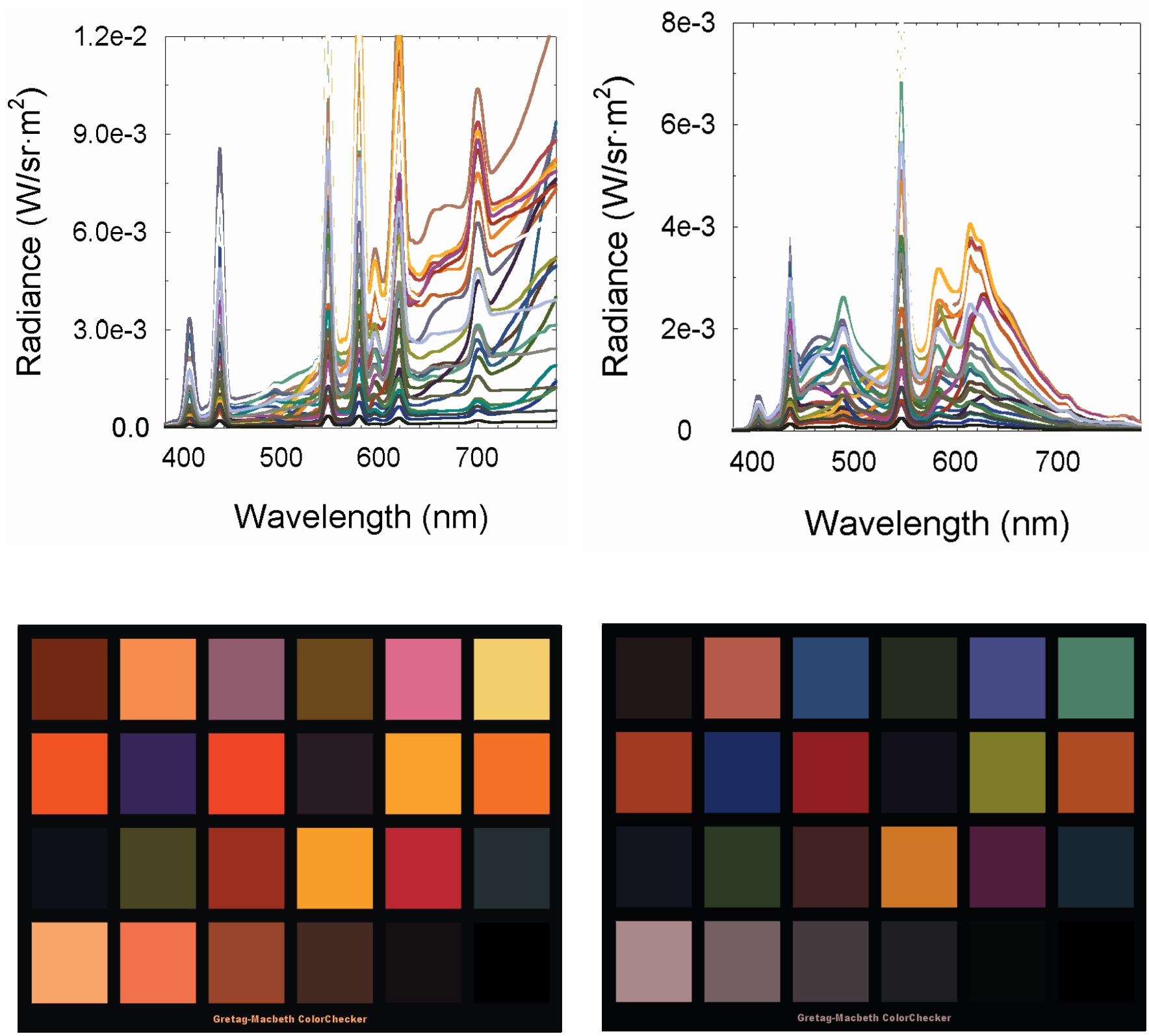

Color Plate 3. Top: Color stimuli of the ColorChecker Chart under metal halide light source (HWL lamp). Bottom: Simulation of the image captured by our digital image capture device. (Francisco Martínez-Verdú, et al, pp.279-295).

Color Plate 4. Top: Color stimuli of the ColorChecker Chart under fluorescent light source (DAY lamp). Bottom: Simulation of the image captured by our digital image capture device. (Francisco Martínez-Verdú, et al, pp.279-295). 

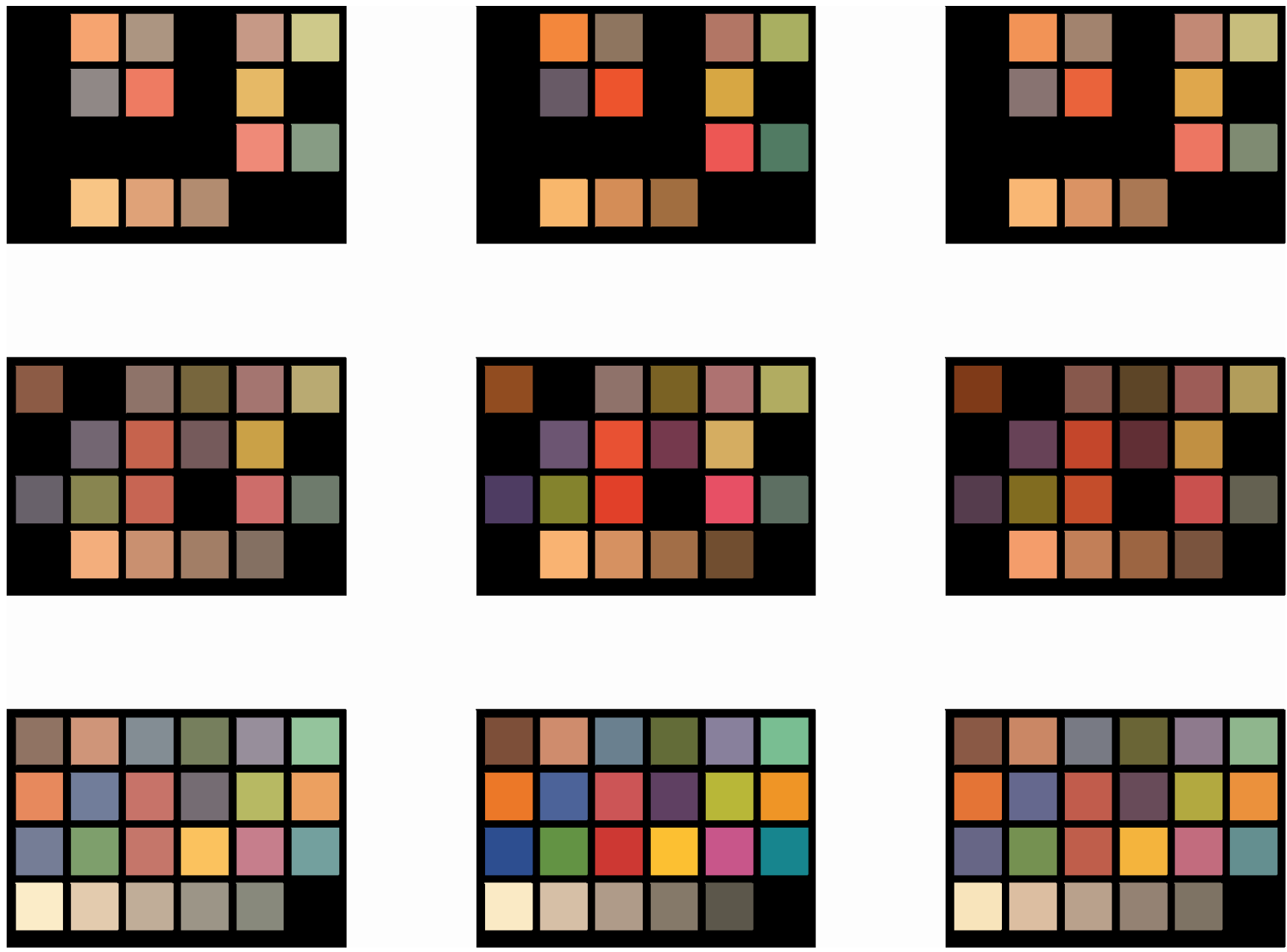

Color Plate 5. SRGB simulation of the ColorChecker chart captured by our color device under three light sources (top: incandescent lamp with $N=4$; center: metal halide lamp with $N=5.6$; bottom: fluorescent daylight lamp with $N=5.6$ ). Left: raw reproduction model; center: original CIE-XYZ encoding; right: corrected reproduction model. (Notice that the original reproduction level of these colors may have undergone alterations even though the color management algorithms have been performed correctly.) (Francisco Martínez-Verdú, et al, pp.279-295). 